\title{
Energy Procurement Strategies in the Presence of Intermittent Sources
}

\author{
Jayakrishnan Nair \\ Electrical Engineering, California Institute of Technology, Pasadena, CA, 91125, ujk@caltech.edu \\ Sachin Adlakha \\ Center for Mathematics of Information, California Institute of Technology, Pasadena, CA, 91125, adlakha@caltech.edu \\ Adam Wierman \\ Computing and Mathematical Sciences, California Institute of Technology, Pasadena, CA, 91125, adamw@caltech.edu

\begin{abstract}
The increasing penetration of intermittent, unpredictable renewable energy sources, e.g., wind energy, poses significant challenges for utility companies trying to incorporate renewable energy in their portfolio. In this work, we study the problem of conventional energy procurement in the presence of intermittent renewable resources. We model the problem as a variant of the newsvendor problem, in which the presence of renewable resources induces supply side uncertainty, and in which conventional energy may be procured in three stages to balance supply and demand. We compute closed form expressions for the optimal energy procurement strategy and study the impact of increasing renewable penetration, and of proposed changes to the structure of electricity markets. We explicitly characterize the impact of a growing renewable penetration on the procurement policy by considering a scaling regime that models the aggregation of unpredictable renewable sources. A key insight from our results is that there is a separation between the impact of the stochastic nature of this aggregation, and the impact of market structure and forecast accuracy. Additionally, we study two proposed changes to the market structure: the addition and the placement of an intermediate market. We show that addition of an intermediate market does not necessarily increase the efficiency of utilization of renewable sources. Further, we show that the optimal placement of the intermediate market is insensitive to the level of renewable penetration.
\end{abstract}

\section{Introduction}

Society's insatiable appetite for energy and growing environmental concerns have led many states in the United States to enact renewable portfolio standards. The standards mandate that utility companies must procure a certain percentage of their electricity from renewable sources (Wiser 2008). For example, California has set the goal that $33 \%$ of its electricity should come from renewable sources by 2020. Among possible renewable sources, wind energy is expected to play a major role. There has been an explosive growth in installed wind capacity over the last few years (Global Wind Energy Council 2009) due to the ease of installation, as well as the low maintenance and operational costs. 
Wind energy, and renewable energy in general, ${ }^{1}$ pose a number of significant challenges as a result of the intermittency and the unpredictability of the available energy. In particular, in order to maintain balanced operation of the grid, a suitable amount of 'reserve' capacity of conventional generation must be procured to absorb the volatility created by the uncertainty in wind generation.

However, current electricity markets that govern energy procurement were designed for a scenario where there is very little uncertainty. More specifically, until now, supply side uncertainty has been low, and mainly arises due to generator failures, which are typically rare. Furthermore, accurate demand forecasting ensures that the uncertainty in demand is small. However, going forward, the introduction of large volumes of highly intermittent and unpredictable renewable generation will increase supply side uncertainty dramatically. Thus, incorporating wind energy into the energy portfolio of utilities is a challenging task that requires rethinking the current design of electricity markets (GE Energy 2010, GE Energy Consulting 2007). This paper seeks to provide insights into the impact of increasing supply side uncertainty on the 'efficiency' of procurement in electricity markets.

Utility companies typically purchase their electricity via two modes of operation - bilateral long term contracts and competitive electricity markets (Kirschen and Strbac 2004, Stoft 2002, Harris 2006). In the former, utility companies sign long term bilateral contracts with various generators to supply certain amounts of electricity for specified periods. Currently, most utility companies purchase the bulk of their generation through long term bilateral contracts. This is feasible because the aggregate demand is highly predictable and because most conventional generators have very little uncertainty. To account for daily (or hourly) fluctuations in demand, the utility companies purchase the remainder of their electricity in competitive electricity markets. In such markets, buyers (i.e., utility companies) and sellers (generators) participate in a single market that is run by a third party called the independent service operator (ISO). These markets typically consist of day-ahead (DA) forward market as well as a real time (RT) market. A utility company may buy electricity in both markets in order to ensure that it has enough supply to meet the demand.

Integration of wind energy into current electricity markets has attracted considerable interest in recent years (DeMeo et al. 2007, Negrete-Pincetic et al. 2010). Broadly speaking, there are two different approaches to integrate wind into current electricity markets. In one approach, wind power producers participate only in competitive electricity markets (Bitar et al. 2011), e.g., California's

\footnotetext{
${ }^{1}$ For the remainder of the paper, we will use wind energy interchangeably with renewable energy; the models and the insights of our paper apply to any form of intermittent resource.
} 
participating intermittent resource program (PIRP). In the second approach, the wind power producers do not participate in electricity markets; instead, they sign long term multi-year contracts with utility companies. In such contracts, the utility company acquires rights to the energy generated from a certain wind farm installation in return for a predetermined payment. For example, Southern California Edison (SCE), a major utility company serving the greater Los Angeles area, has signed various contracts spanning 2-10 years with various wind farms to procure wind energy ranging from 66.6 MW to 115 MW (Southern California Edison 2011).

In this paper, we study the consequences of following the second approach. In particular, we study a setting where utility companies have procured large volumes of intermittent, unpredictable renewable energy via long term contracts. Because the realized amount of wind energy is variable and unpredictable, the utility company must still procure conventional generation via the electricity markets. Our goal is to study the impact of this long term commitment to renewable generation on the procurement strategies for conventional generation.

Given the complexity of electricity markets, we must consider a simplified model to be able to obtain analytical results. To that end, we consider a setting that ignores many complexities of generation and transmission (e.g., ramp constraints and line capacities), but models explicitly the multi-timescale nature of electricity markets. That is, we model real time, day ahead, and long term markets, assuming that the electricity price is larger in markets closer to real time, but that the accuracy of predictions of the available renewable energy is also better in markets closer to real time. The details of our model can be found in Section 2 .

The main contributions of the work fall, briefly, into two categories: (i) we characterize the optimal procurement strategy in the presence of long-term contracts for intermittent, unpredictable generation; and (ii) we study the impact of increasing renewable penetration as well as possible changes to market structure on the optimal procurement strategy. We describe these each in more detail in the following.

The first contribution of this work is to characterize the optimal procurement strategy for a utility company that has a long term contract with an unpredictable generation source, e.g., wind energy (see Section 3). More specifically, we derive closed-form formulas for the optimal procurement that a utility company needs to make in both long term and day ahead markets. This solution is a generalization of solutions for the classical newsvendor problem (Arrow et al. 1951, Silver et al. 1998, Khouja 1999). A key feature of our result is that the optimal procurement quantities can be viewed in terms of reserves, where these reserve quantities are the additional purchases that the 
utility company needs to make to balance the current uncertainty of the supply and the higher cost of procuring energy in future markets.

In addition to deriving the optimal procurement strategy, we are able to study how it changes as the penetration of renewable energy grows (see Section 4). In particular, we consider a scenario where the quantity of renewable generation contracted for grows, and ask how the procurement changes. The scaling for increasing penetration that we consider allows for a wide variety of models for how the unpredictability of renewable generation changes with increased penetration. For example, it includes scaling via additional sources with either independent or highly correlated generation. Our main result from this section yields an extremely simple, informative, manageriallevel equation summarizing the impact of renewable generation on the procurement of conventional generation. Specifically, Theorem 2 states that the average total procurement of conventional generation in the presence of a long term contract for wind is

$$
d-\alpha \gamma+\delta \gamma^{\theta}
$$

where $d$ is the demand, $\alpha$ is the generation of average output of a single wind farm, $\gamma$ is the number of wind farms (i.e., the renewable penetration), $\theta$ is a constant capturing the dependence between the generations of different wind farms, and $\delta$ is a constant that depends on the details of the market structure. The way to interpret this equation is as follows. $d-\alpha \gamma$ represents the minimum average procurement, since this is the amount of demand that is not met by the wind. Thus, the 'extra' generation required because of the uncertainty of the wind is $\delta \gamma^{\theta}$. The key point about this term is that $\gamma^{\theta}$ is purely dependent on the degree of renewable penetration and the correlation between renewable sources; thus the impact of market structure is limited to $\delta$.

There are two types of changes to the market structure that are most commonly considered: changing the placement of the day ahead market, e.g., by moving it closer to real time; and adding markets, e.g., adding a new market between the day ahead and real time markets (Rajagopal et al. 2012). The results in Sections 5 and 6 address each of these possibilities.

First, Section 5 studies the optimal placement of markets. This is a particularly salient issue because one might expect that as the penetration of renewable energy increases, it is beneficial to shift markets closer to real time, in order to take advantage of the improved prediction accuracy of the renewable generation. Our results highlight that this intuition may not be true. Specifically, we prove that, under very general assumptions, the placement of the day ahead market that minimizes the average total cost of procurement is independent of the penetration of renewables (Theorem 3). 
Next, Section 6 studies the impact of additional markets on procurement. The addition of markets is often suggested as a way to help incorporate renewable generation by providing new markets closer to real time where predictions about renewable availability are more accurate. Our results highlight that one needs to be careful when considering such a change. Specifically, we contrast procurement in a two level market with procurement in a three level market in order to understand the role of adding an intermediate market. Of course, the cost of procurement always decreases as additional markets are introduced. However, with environmental concerns in mind, the key question is not about cost but about the amount of conventional generation procured. Perhaps surprisingly, additional markets do not always reduce the amount of conventional generation procured. Specifically, if we consider the addition of an intermediate market, then the average amount of conventional generation may drop or grow depending on the quality of the estimates for renewable generation, i.e., $\delta$ in Equation (1) may decrease or increase. Informally, if the estimation error is, in a sense, light-tailed (e.g., Gaussian), then the addition of an intermediate market reduces procurement of conventional generation (Theorem 4); but if the error has a heavy-tail (e.g., a power-law), then the addition of an intermediate market can have the opposite effect (Lemma 2).

Finally, it is important to note that though we focus entirely on procurement of electricity, our model and results should be of interest more broadly within the domain of inventory theory. Specifically, at its core, our model considers a three level newsvendor problem, and characterizes the impact on procurement in a setting in which uncertainty grows.

The remainder of the paper is organized as follows. Section 2 describes our market model as well as the model for wind forecast evolution. The optimal procurement strategy for three markets is derived in Section 3. We study the effect of increasing wind penetration in Section 4. Section 5 shows that the optimal placement of the intermediate market is insensitive to the capacity of wind generation. In Section 6, we study the impact of additional markets on the total conventional procurement. Section 7 describes related work and Section 8 concludes the paper.

\section{Market model}

Our goal in this paper is to understand how the presence of long term contracts for intermittent, unpredictable renewable generation impacts the procurement of conventional generation; and the role the structure of the electricity market plays. Thus, at the core of the paper, is a model for the electricity markets, which we describe in this section. The model we study is necessarily simple, so as to facilitate analysis and allow the derivation of simple and informative closed-form characterizations of procurement. 
The key feature we seek to capture is the multi-timescale nature of electricity markets. Thus, we ignore issues such as generator ramping constraints and transmission network capacity constraints. Furthermore, we assume that the utility company has no access to large scale storage capacity. These assumptions allow us to focus on a single instant of time, which we denote by $t=0$, and consider only aggregate supply and demand. We assume that the demand (denoted by $d$ ) is fixed and known ahead of time. ${ }^{2}$

There are two key components of our model of the electricity markets: (i) the model of the structure of the markets; and (ii) the model of forecast evolution for the renewable generation realized at time $t=0$.

Market structure. Recall that the setting we consider in this paper is that of a utility company with long term contracts for uncertain, intermittent, renewable energy. Given such a long term contract, the utility company participates in a three tiered market for conventional generation consisting of:

(i) a long term market, in which a purchase commitment is made at time $-T_{l t}$ and the price of energy is $p_{l t}$,

(ii) an intermediate market (typically day ahead), in which a purchase commitment is made at time $-T_{i n}$ and the price of energy is $p_{i n}$,

(iii) a real time market, in which purchase commitment is made at time $t=0$ and the price of energy is $p_{r t}$,

with $-T_{l t}<-T_{i n}<0$. In practice, the long term market could take place years or months ahead of time $t=0$ and the intermediate market could take place a day or several hours ahead of time $t=0$.

We assume that the energy prices in the markets are fixed, known ahead of time, and ordered such that $0<p_{l t}<p_{i n}<p_{r t}$. In other words, we assume that utility companies are price-takers in these markets and that conventional energy gets more expensive closer to real time. The ordering of prices is a mild assumption and is borne out of the fact that any conventional energy that is demanded closer to real time is provided by generators that have low start up time. Typically these generators are more expensive than the generators that require several hours to start up. Furthermore, in practice, the price of electricity in real time markets tends to be higher on average than in day ahead markets.

\footnotetext{
${ }^{2}$ Demand-side uncertainty can be folded into the supply-side uncertainty in our model.
} 
Renewable forecasts. A key feature of the model is how we capture the uncertainty associated with the availability of renewable generation. Clearly, the procurement policy of a utility company will depend upon the information available to it at the time of each market. In particular, at the time of each market, the utility company needs to forecast how much wind generation will be available at time $t=0$. Note that if the available wind energy was known with certainty, the utility company could purchase its entire remaining demand exactly in the long term market; however because the amount of available wind energy at time $t=0$ is highly uncertain at the time of the long term market, the utility company needs to balance its lowest cost purchase in long term with better estimates of wind energy closer to real time. To capture this, we consider a model where the forecast accuracy of the wind generation improves upon moving closer to real time, i.e., $t=0$.

Let $w$ denote the actual wind energy that is available at time $t=0$. We assume that the utility company comes to know the value of $w$ at time $t=0$. Let $\hat{w}_{l t}$ and $\hat{w}_{i n}$ denote respectively the forecasts of $w$ available at the time of the long term market (i.e., at $t=-T_{l t}$ ) and the intermediate market (i.e., at $t=-T_{i n}$ ). Formally, we assume that

$$
\hat{w}_{i n}=\hat{w}_{l t}-\mathcal{E}_{1}, \text { and } w=\hat{w}_{i n}-\mathcal{E}_{2},
$$

where $\mathcal{E}_{1}$ and $\mathcal{E}_{2}$ are zero mean independent random variables. In other words, we assume that the forecast of $w$ evolves with independent increments. The random variables $\mathcal{E}_{1}$ and $\mathcal{E}_{2}$ capture the evolution of the forecast of $w$ between the instants of the three markets. Note that $\hat{w}_{l t}$ is a coarser estimate of $w$ than $\hat{w}_{i n}$, since the long term forecast error $\hat{w}_{l t}-w=\mathcal{E}_{1}+\mathcal{E}_{2}$ is more variable than the intermediate forecast error $\hat{w}_{i n}-w=\mathcal{E}_{2}$. A special case of our forecast evolution model is the well studied martingale model of forecast evolution (Hausman 1969, Graves et al. 1985, Heath and Jackson 1994), in which it is additionally assumed that $\mathcal{E}_{1}$ and $\mathcal{E}_{2}$ follow a Gaussian distribution.

Let $\left[L_{1}, R_{1}\right]$ and $\left[L_{2}, R_{2}\right]$ denote respectively the supports of the random variables $\mathcal{E}_{1}$ and $\mathcal{E}_{2} .{ }^{3}$ We make the following regularity assumptions on the distributions of $\mathcal{E}_{1}$ and $\mathcal{E}_{2}$ : they are associated with continuously differentiable density functions, denoted by $f_{\mathcal{E}_{1}}(\cdot)$ and $f_{\mathcal{E}_{2}}(\cdot)$ respectively, with $f_{\mathcal{E}_{i}}(x)>0$ for $x \in\left(L_{i}, R_{i}\right)$.

In the remainder of this paper, we use the following notation. We use $P(E)$ to denote the probability of an event $E$, and $\bar{F}_{X}$ to denote the complementary cumulative distribution function associated with the random variable $X$, i.e., $\bar{F}_{X}(x)=P(X>x)$. Finally, $[x]_{+}:=\max \{x, 0\}$.

${ }^{3} L_{1}, L_{2} \in\{-\infty\} \cup \mathbb{R}$ and $R_{1}, R_{2} \in \mathbb{R} \cup\{\infty\}$. 


\section{Optimal procurement}

Given the model in the previous section, we can now pose the procurement problem for the utility company, which is the focus of this paper. As we have described, the objective of the utility company is to procure enough conventional energy to meet its residual demand (i.e., demand minus the available wind energy) at real time. Recall that the cost of procurement is lowest in the long term market. However, the utility company has a very coarse estimate of the available wind energy at that time. As we move closer to real time, the utility company gets an improving estimate of the available wind energy and hence, of the residual demand. However, conventional energy also gets more expensive as we approach real time. Thus, in order to minimize its procurement cost, the utility company needs to balance the improving forecast accuracy with an increasing cost of procurement approaching real time.

In this section, we formalize the utility's procurement problem, and then characterize the optimal procurement strategy. This strategy is the basis for the explorations of the impact of increased penetration of renewables and changes to market structure that make up the remainder of the paper.

The procurement problem. To begin, note that the procurement decision of the utility in each market can depend only on the information available to the utility company at that time. Specifically, in each market, we consider procurement strategies that depend only on (i) the wind estimate available at the time of purchase, and (ii) the total conventional generation that has already been procured. ${ }^{4}$ Accordingly, let $q_{l t}\left(\hat{w}_{l t}\right)$ denote the quantity of conventional generation procured in the long term market, given the long term wind estimate $\hat{w}_{l t}$. Similarly, let $q_{i n}\left(\hat{w}_{i n}, q_{l t}\right)$ denote the quantity of conventional generation procured in the intermediate market, given the corresponding wind estimate $\hat{w}_{i n}$ and $q_{l t}$ (the quantity procured already). Finally, let $q_{r t}\left(w, q_{l t}+q_{i n}\right)$ denote the the quantity of conventional generation procured in the real time market, which depends on the realized wind $w$, and the quantity procured already, i.e., $q_{l t}+q_{i n}$. For notational convenience we often drop the arguments from these functions and simply write $q_{l t}, q_{i n}, q_{r t}$. We make the assumption that the utility cannot sell power in any market ${ }^{5}$, and thus the quantities procured in all three markets must be non negative.

When determining these procurement quantities, we assume that the utility company is seeking to minimize its expected total cost while ensuring that the total quantity purchased in these three

\footnotetext{
${ }^{4}$ In our model, we assume that the estimate of the wind in the real time evolves with independent increments. Hence, it is easy to show that there is no loss of optimality in restricting policies to this class.

${ }^{5}$ Our model can be extended to relax this assumption. However, we do not consider this generalization because it adds complexity with no additional insights.
} 
markets satisfies the residual demand (i.e., the demand minus the available wind in real time). Thus, we can express the optimal procurement problem as follows:

$$
\begin{gathered}
\min _{q_{l t}, q_{i n}, q_{r t}} \mathbb{E}\left[p_{l t} q_{l t}+p_{i n} q_{i n}+p_{r t} q_{r t}\right] \\
\text { subject to } q_{l t} \geq 0, q_{i n} \geq 0, q_{r t} \geq 0 \\
q_{l t}+q_{i n}+q_{r t}+w \geq d .
\end{gathered}
$$

Recall that $q_{l t}, q_{i n}$ and $q_{r t}$ are functions that depend on the corresponding wind estimate and the total procurement so far.

The optimal procurement problem posed above is mathematically equivalent to a variant of the classical newsvendor problem (Khouja 1999, Porteus 2002). In Section 7, we discuss the relationship between our work and the literature on the newsvendor problem.

A final comment about the the optimal procurement problem above is that we have considered a three tiered market structure that models the common current practice. In general, the optimal procurement problem is simply a Markov decision process, and can be studied for arbitrary numbers of tiers, e.g., see Rajagopal et al. (2012). We limit ourselves to a three tiered structure in this paper to keep the analysis simple and the resulting formulas interpretable. Moreover, our formulation allows us to study the impact of increased wind penetration and market structure on the optimal procurement policy, something that is not previously considered in the literature.

Optimal procurement strategy. Given the procurement problem introduced above, we can now study the optimal procurement strategy. The following theorem is the foundational result for the remainder of the paper. It characterizes the optimal procurement strategy for a utility company in a three tiered market.

THEOREM 1. The optimal procurement strategy for the utility company in the three tiered market scenario of Problem $(P)$ is:

$$
\begin{aligned}
q_{l t}^{*} & =\left[d-\hat{w}_{l t}+r_{l t}\right]_{+}, \\
q_{i n}^{*} & =\left[d-\hat{w}_{i n}-q_{l t}+r_{i n}\right]_{+}, \\
q_{r t}^{*} & =\left[d-w-\left(q_{l t}+q_{i n}\right)\right]_{+} .
\end{aligned}
$$

where

$$
r_{i n}=\bar{F}_{\mathcal{E}_{2}}^{-1}\left(\frac{p_{i n}}{p_{r t}}\right)
$$

and $r_{l t}$ is the unique solution of

$$
h(r) \triangleq p_{l t}-p_{i n} \bar{F}_{\mathcal{E}_{1}}\left(r-r_{i n}\right)-p_{r t} P\left(\mathcal{E}_{1}+\mathcal{E}_{2}>r, \mathcal{E}_{1} \leq r-r_{i n}\right)=0 .
$$


The proof of Theorem 1 is included in Appendix A. A key feature of this theorem is that the structure of the the optimal procurement strategy gives a natural interpretation to $r_{l t}$ and $r_{i n}$ as reserve levels. Specifically, at the time of purchase in the long term market, $d-\hat{w}_{l t}$ can be interpreted as an estimate of the conventional procurement that is required to meet the demand. Then $r_{l t}$ is the additional 'reserve' purchased by the utility to balance the current wind uncertainty and the higher cost of conventional energy in subsequent markets. The reserve $r_{i n}$ has a similar interpretation. We will henceforth refer to $r_{l t}$ and $r_{i n}$ as the (optimal) long term and intermediate reserves respectively.

It is important to point out that the reserves $r_{l t}$ and $r_{i n}$ may be positive or negative. A negative reserve implies that it is optimal for the utility to maintain a net procurement level that is less than the currently anticipated residual demand, and purchase any shortfall in subsequent markets. The values of the optimal reserves depend on the prices of conventional energy in the three markets and the distributions of the random variables $\mathcal{E}_{1}$ and $\mathcal{E}_{2}$, which capture the accuracy of the wind forecasts $\hat{w}_{l t}$ and $\hat{w}_{i n}$. Note that the optimal reserves do not depend on the demand $d$, or on procurements made in prior markets.

As is evident from Equation (6), the optimal reserve in the intermediate market is the $p_{i n} / p_{r t^{-}}$ th quantile of the complementary cumulative distribution function associated with the random variable $\mathcal{E}_{2}$. This solution is structurally similar to the the critical fractile solution of the classical news vendor model (Arrow et al. 1951). This is because, at the time of the intermediate market, the utility company (having already purchased the quantity $q_{l t}$ ) faces a problem similar to the classical news vendor problem.

Finally, Theorem 1 highlights the need for the following additional assumption in order to avoid trivial solutions.

Assumption 1. We assume that the demand is large enough that the utility company procures a positive quantity in the long term market. That is, $d-\hat{w}_{l t}+r_{l t}>0$.

This assumption ensures that the optimal procurement problem in the three market has a nontrivial solution in the following sense: if $d-\hat{w}_{l t}-r_{l t} \leq 0$, then the utility company procures $q_{l t}^{*}=0$ in the long term. In this case, the procurement problem effectively reduces to a two market scenario. Intuitively, this assumption will hold as long as the demand $d$ exceeds the peak capacity of the renewable installations, which is of course true in most current practical scenarios. The benefit of Assumption 1 is that it allows us to rewrite the optimal procurement quantities in three markets as follows:

$$
q_{l t}^{*}=d-\hat{w}_{l t}+r_{l t}
$$




$$
\begin{aligned}
q_{i n}^{*} & =\left[\mathcal{E}_{1}-r_{l t}+r_{i n}\right]_{+}, \\
q_{r t}^{*} & =\left[\mathcal{E}_{2}-r_{l t}+\min \left\{\mathcal{E}_{1}, r_{l t}-r_{i n}\right\}\right]_{+},
\end{aligned}
$$

where we abuse notation to let $q_{l t}^{*}, q_{i n}^{*}$ and $q_{r t}^{*}$ represent the (random) quantities that are purchased in the long term, the intermediate, and the real time markets respectively.

\section{The impact of increasing renewable penetration}

The penetration of renewable energy, in particular of wind energy, is poised for major growth over the coming years. A consequence of this is that utility companies will face an ever increasing supply side uncertainty. In this section, we explore the impact of this growth on procurement. Specifically, we ask: how will the optimal procurement policy change as the volume of intermittent wind resources increases?

In this section, we introduce a scaling regime for wind penetration, which models the effect of aggregating the output of several wind generators. A key feature of our scaling model is that it allows for varying levels of stochastic dependence between the intermittent energy sources being aggregated. For example, our model lets us study the aggregation of independent sources, as well as perfectly correlated sources. Based on this scaling model, we study how the optimal reserves, the amount of conventional generation procured, as well as the cost of procurement scale with increasing wind penetration. Our analysis yields clean and easy to interpret scaling laws for these quantities. Remarkably, the scaling laws reveal a decoupling between the impact of the level of stochastic dependence between different wind sources and the impact of market structure and wind forecast accuracy.

\subsection{Scaling regime}

We begin by describing our scaling model for wind penetration. We start with a baseline scenario. Let us denote by $\alpha$ the average output of a single intermittent generator. For concreteness, we refer to this as the baseline wind farm in the following. We let $\tilde{\mathcal{E}}_{1}$ and $\tilde{\mathcal{E}}_{2}$ be the error random variables that relate the long term wind estimate $\hat{w}_{l t}$ to the estimate of the wind in the intermediate market $\hat{w}_{\text {in }}$ and the actual wind realization $w$ (see Equation (2)). We assume that in the long term market there is no better estimate of the wind than the long term average, i.e., $\hat{w}_{l t}=\alpha$. Note that the optimal procurement in the baseline scenario (before scaling) can be computed using Theorem 1. For the remainder of this section, we use $\tilde{r}_{l t}$ and $\tilde{r}_{i n}$ to denote respectively the optimal long term and the intermediate reserve in the baseline scenario. 
To scale the wind penetration, we introduce a scale parameter $\gamma$ that is proportional to the capacity of wind generation. The scale parameter $\gamma$ may be interpreted as the number of (homogeneous) wind farms whose aggregate output is available to the utility company. Thus, the average wind energy available to the utility company is given by $\hat{w}_{l t}(\gamma)=\gamma \alpha$. Clearly, as the capacity of wind generation scales, the error in the wind forecast available at both the long term and the intermediate markets will also scale, and this scaling will depend on the correlation between the generation at each of the wind farms. We use the following simple, but general scaling model.

$$
\begin{aligned}
& \hat{w}_{l t}(\gamma)=\gamma \alpha, \\
& \mathcal{E}_{1}(\gamma)=\gamma^{\theta} \tilde{\mathcal{E}}_{1}, \\
& \mathcal{E}_{2}(\gamma)=\gamma^{\theta} \tilde{\mathcal{E}}_{2},
\end{aligned}
$$

where we let $\theta \in[1 / 2,1]$.

To interpret this scaling, consider first the case $\theta=1$. In this case, $\mathcal{E}_{1}=\gamma \tilde{\mathcal{E}}_{1}$ and $\mathcal{E}_{2}=\gamma \tilde{\mathcal{E}}_{2}$, implying that $\hat{w}_{i n}(\gamma)$ and $w(\gamma)$ scale proportionately with $\gamma$. This scaling corresponds to a scenario where the aggregate wind output with $\gamma$ wind farms is simply $\gamma$ times the output of the baseline wind farm. One would expect such a scaling to occur if the wind farms that are co-located.

The case of $\theta=1 / 2$ (with $\tilde{\mathcal{E}}_{1}$ and $\tilde{\mathcal{E}}_{2}$ being normally distributed) corresponds to a central limit theorem scaling, and seeks to capture the scenario where the output of each wind farm is independent. Equation (9) captures this scenario exactly if the forecast evolution distributions for each wind farm follow a Gaussian distribution. ${ }^{6}$ If not, Equation (9) can be interpreted as an approximation for large enough $\gamma$ based on the central limit theorem. Intuitively, one would expect such a scaling if the different wind farms are geographically far apart.

Finally, the case $\theta \in\left(\frac{1}{2}, 1\right)$ seeks to capture correlations that are intermediate between independence and perfect correlation (see, for instance Baldovin and Stella (2007), Umarov et al. (2008)).

Note that the forecast error distributions grow slowest when the outputs of different wind farms are independent, and fastest when the outputs are perfectly correlated.

\subsection{Our results}

Given the scaling regime described above, we are now ready to characterize the impact of increasing penetration of intermittent resources on the procurement of conventional generation. First, we analyze how the optimal reserve levels scale with increasing wind penetration. Next, we use these results to obtain scaling laws for the procurement quantities in the three markets, and also the total procurement and the total cost of procurement.

${ }^{6}$ This is a common assumption in the literature, see, Heath and Jackson (1994), Wang et al. (2012), Rajagopal et al. (2012). 
Scaling of reserve levels. The following lemma characterizes the scaling of the optimal reserves under our wind scaling model. It shows the optimal reserve levels in the long term and intermediate markets follow the same scaling as imposed on the distributions of the forecast errors.

LEMma 1. Under the scaling regime defined in Equation (9), the optimal long term and intermediate reserves scale as:

$$
r_{l t}(\gamma)=\gamma^{\theta} \tilde{r}_{l t}, \quad r_{i n}(\gamma)=\gamma^{\theta} \tilde{r}_{i n}
$$

Proof. From Theorem 1, given any scale parameter $\gamma$, the optimal reserve in the intermediate market is the unique solution of the equation

$$
r_{i n}(\gamma)=\bar{F}_{\mathcal{E}_{2}(\gamma)}^{-1}\left(\frac{p_{i n}}{p_{r t}}\right) .
$$

Using the definition of complementary cumulative distribution function and noting that $\mathcal{E}_{2}(\gamma)=$ $\gamma^{\theta} \tilde{\mathcal{E}}_{2}$, we get that the optimal intermediate reserve is given by the unique solution of the equation

$$
P\left(\gamma^{\theta} \tilde{\mathcal{E}}_{2}>r_{i n}(\gamma)\right)=\frac{p_{i n}}{p_{r t}}
$$

It is easy to check that $r_{i n}(\gamma)=\gamma^{\theta} \tilde{r}_{i n}$ satisfies the above equation. Similarly, the optimal long term reserve is the unique solution to the equation

$$
p_{l t}-p_{i n} P\left(\mathcal{E}_{1}(\gamma)>r_{l t}(\gamma)-r_{i n}(\gamma)\right)-p_{r t} P\left(\mathcal{E}_{1}(\gamma)+\mathcal{E}_{2}(\gamma)>r_{l t}(\gamma), \mathcal{E}_{1}(\gamma) \leq r_{l t}(\gamma)-r_{i n}(\gamma)\right)=0
$$

Substituting for $r_{i n}(\gamma)=\gamma^{\theta} \tilde{r}_{i n}$, we note that $r_{l t}(\gamma)=\gamma^{\theta} \tilde{r}_{l t}$ is a unique solution to the above equation.

Scaling of procurement quantities. Using Lemma 1, we can now characterize the optimal procurement amounts in the three markets. As in Section 3, to avoid the trivial solution with zero long term procurement, we restrict the range of scale parameter $\gamma$ to satisfy

$$
d>\gamma \alpha-\gamma^{\theta} \tilde{r}_{l t},
$$

This ensures that the optimal long term procurement, given by

$$
q_{l t}^{*}(\gamma)=\left[d-\hat{w}_{l t}(\gamma)+r_{l t}(\gamma)\right]_{+}
$$

is strictly positive. ${ }^{7}$ For the remainder of this section, we focus on the range of scale parameter $\gamma$ that satisfies Condition (10). Intuitively speaking, Condition (10) will hold as long as the demand

${ }^{7}$ One sufficient $\theta$-independent condition that ensures the inequality is given by $\gamma<\frac{d}{\alpha+\left|\tilde{r}_{l t}\right|}$. This imposes an upper bound on the degree of penetration that we consider. 
is larger than the peak wind capacity. Under this assumption, using Equation (8) and Lemma 1, we get that the optimal procurement quantities in the long term, intermediate, and real time markets is given by

$$
\begin{aligned}
q_{l t}^{*}(\gamma) & =d-\gamma \alpha+\gamma^{\theta} \tilde{r}_{l t}, \\
q_{i n}^{*}(\gamma) & =\gamma^{\theta}\left[\tilde{\mathcal{E}}_{1}-\tilde{r}_{l t}+\tilde{r}_{i n}\right]_{+}, \\
q_{r t}^{*}(\gamma) & =\gamma^{\theta}\left[\tilde{\mathcal{E}}_{2}-\tilde{r}_{l t}+\min \left\{\tilde{\mathcal{E}}_{1}, \tilde{r}_{l t}-\tilde{r}_{i n}\right\}\right]_{+} .
\end{aligned}
$$

The above equations reveal that as we increase the penetration of wind, the optimal procurement in the intermediate and the real time markets increases proportionately to $\gamma^{\theta}$. This increasing procurement in markets closer to real time is a consequence of the increasing uncertainty in the renewable forecasts. Indeed, note that the procurements in the intermediate and real time markets scale in exactly the same manner as the forecast error distributions. Therefore, these procurements scale slowest when outputs of different wind farms are independent, and fastest when the outputs are perfectly correlated. From the standpoint of the system operator, Equation (11) describes how the installed capacity of fast ramp conventional generators that can supply energy in the intermediate and real time markets needs to scale as the capacity of wind generation scales.

Scaling of total procurement and total cost of procurement. As we scale the wind capacity, we expect that the amount of total conventional energy procured, as well as the cost of procurement must decrease. The following theorem characterizes the scaling laws for these quantities. Let $T P(\gamma)$ and $T C(\gamma)$ denote respectively the total procurement of conventional energy and the total cost of procurement, corresponding to scale parameter $\gamma$.

THEOREM 2. For the range of scale parameter $\gamma$ satisfying Equation (10), the expected total procurement and the expected total cost are given by

$$
\begin{aligned}
& \mathbb{E}[T P(\gamma)]=d-\alpha \gamma+\delta \gamma^{\theta}, \\
& \mathbb{E}[T C(\gamma)]=p_{l t}(d-\alpha \gamma)+\delta^{\prime} \gamma^{\theta}
\end{aligned}
$$

where, $\delta \geq 0$ and $\delta^{\prime} \geq 0$ are defined as

$$
\begin{aligned}
& \delta \triangleq \tilde{r}_{l t}+\mathbb{E}\left[\tilde{\mathcal{E}}_{1}-\left(\tilde{r}_{l t}-\tilde{r}_{i n}\right)\right]_{+}+\mathbb{E}\left[\tilde{\mathcal{E}}_{2}-\tilde{r}_{l t}+\min \left\{\tilde{\mathcal{E}}_{1}, \tilde{r}_{l t}-\tilde{r}_{i n}\right\}\right]_{+}, \\
& \delta^{\prime} \triangleq p_{l t} \tilde{r}_{l t}+p_{i n} \mathbb{E}\left[\tilde{\mathcal{E}}_{1}-\left(\tilde{r}_{l t}-\tilde{r}_{i n}\right)\right]_{+}+p_{r t} \mathbb{E}\left[\tilde{\mathcal{E}}_{2}-\tilde{r}_{l t}+\min \left\{\tilde{\mathcal{E}}_{1}, \tilde{r}_{l t}-\tilde{r}_{i n}\right\}\right]_{+} .
\end{aligned}
$$


The scaling law (12) has the following interpretation. If there was no uncertainty in the wind generation, i.e., $w(\gamma)=\hat{w}_{l t}(\gamma)=\alpha \gamma$, then the utility would procure the exact residual demand, i.e., $d-\alpha \gamma$ in the long term market. This is the minimum possible value of expected total procurement. From (12), we see that on average, the utility has to make an 'additional procurement' equal to $\delta \gamma^{\theta}$ as a result of the uncertainty in the wind generation. The fact that this additional procurement grows proportionately to $\gamma^{\theta}$ highlights the benefit of aggregating independent wind sources. Specifically, if $\theta=1$, i.e., the wind farms are co-located, then the additional procurement is of the same order as the wind capacity. On the other hand, if $\theta=1 / 2$, i.e., the wind farms are geographically far apart, then the additional procurement grows much slower than the wind capacity.

The scaling law (13) has a similar interpretation. Note that $p_{l t}(d-\gamma \alpha)$ is the minimum possible value of expected cost of procurement for the utility company; this would be the case if there was no uncertainty in the wind generation. From (13), we see that on average, the utility incurs an 'additional cost' equal to $\delta^{\prime} \gamma^{\theta}$ as a result of the uncertainty in the wind generation. Once again, we see the benefit of aggregating independent wind sources. Thus, intuitively speaking, a utility company would benefit from signing long contracts with wind generators that are as geographically spread out as possible.

The structure of the scaling laws (12) and (13) also reveals an interesting separation between the impact of the level of stochastic dependence between different wind sources and the impact of market structure and wind forecast accuracy. To see this, consider the 'additional procurement' $\delta \gamma^{\theta}$ in Equation (12) and the 'additional cost' $\delta^{\prime} \gamma^{\theta}$ in Equation (13). The factor $\gamma^{\theta}$ is purely dependent upon the volume of wind capacity and the stochastic dependence between the outputs of the different wind farms. On the other hand, the factors $\delta$ and $\delta^{\prime}$ depend on only the price of conventional energy in the three markets, and the baseline forecast error distributions. In other words, $\delta$ and $\delta^{\prime}$ are invariant with respect to the aggregation of wind sources. As we discuss in the next section, this separation has interesting consequences for market design.

We now present the proof of Theorem 2, which follows easily from Lemma 1 and Theorem 1.

Proof of Theorem 2. The expected total procurement and the expected total cost follow trivially from Equation (11). To prove that $\delta \geq 0$, we note that the optimization problem $P$ requires that the total procurement exceed $d-w(\gamma)$ for every realization of wind $w$. Thus, the expected total procurement must satisfy

$$
\mathbb{E}[T P(\gamma)] \geq d-\gamma \alpha
$$

where we use the fact that the expected value of $w(\gamma)$ is $\gamma \alpha$. From Equation (12), we get that $\delta \geq 0$. Finally, note that $\delta^{\prime}$ can be written as

$\delta^{\prime}=p_{l t} \delta+\left(p_{i n}-p_{l t}\right) \mathbb{E}\left[\mathcal{E}_{1}(1)-\left(r_{l t}(1)-r_{i n}(1)\right)\right]_{+}+\left(p_{r t}-p_{l t}\right) \mathbb{E}\left[\mathcal{E}_{2}(1)-r_{l t}(1)+\min \left\{\mathcal{E}_{1}(1), r_{l t}(1)-r_{i n}(1)\right\}\right]_{+}$. 
Since the prices satisfy $0<p_{l t}<p_{i n}<p_{r t}$ and $\delta \geq 0$, we get that $\delta^{\prime} \geq 0$. This proves the theorem.

\section{The optimal placement of the intermediate market}

In current electricity markets, intermediate markets take place about 14 hours before $t=0$ (these markets are called day ahead or forward markets) (CAISO 2011). Because the demand can be predicted reasonably accurately at that time, this allows the utility company to procure most of their generation much before the time of use. However, accurate prediction of wind in the current day ahead markets is not feasible. Thus, it is commonly suggested that as the penetration of wind increases, the system operator may decide to move the intermediate market closer to real time to allow for better prediction of the wind. In this section, we consider the optimal placement of the intermediate market and study how this optimal placement changes as we increase the penetration of wind energy.

Recall that, in our model, the intermediate market takes place $T_{i n}$ time units prior to the time of use (i.e. $t=0)$. If the system operator were to move this intermediate market closer to real time, this would imply better estimates of available wind at that time. However, in moving the market closer to real time, the procured conventional generation must come from generators that have faster ramp up times. These generators are typically more expensive and hence we would expect that the price of the conventional generation in the intermediate market would increase as we move the market closer to real time. We define the optimal placement of the intermediate market as the one that minimizes the average total procurement cost. Note that this optimal placement would balance an improving forecast of the wind generation moving closer to real time with an increasing price of conventional energy.

The optimal placement is clearly a function of how the forecast errors $\mathcal{E}_{1}$ and $\mathcal{E}_{2}$ change as the function of the intermediate market time $T_{i n}$. It also depends on how the price of the conventional generation in the intermediate market $p_{i n}$ changes as the $T_{i n}$ changes. Let us denote by $T_{i n}^{*}$ the optimal placement of the intermediate market that minimizes the expected total cost of procuring conventional generation (note that the market itself would be at time $-T_{i n}^{*}$ ).

One question of interest from the system operator viewpoint is how does the optimal placement of the intermediate market change as we increase the penetration of wind energy? The answer to this question has significant implications for future market design. Remarkably, based on the scaling regime developed in the previous section, the following theorem tells us that the optimal placement does not depend upon the scale parameter $\gamma$ or the aggregation parameter $\theta$. 
TheOREM 3. For the wind scaling satisfying Equation (10), over the range of $\gamma$ satisfying Condition (10), the optimal placement of the intermediate market is independent of the scale parameter $\gamma$ and the correlation parameter $\theta$.

Proof. From Theorem 2, we note that the expected total cost of procurement is given by

$$
\mathbb{E}[T C(\gamma)]=p_{l t}(d-\alpha \gamma)+\delta^{\prime} \gamma^{\theta}
$$

Furthermore, the effect of placement is only on the parameter $\delta^{\prime}$ (via the distributions $\tilde{\mathcal{E}}_{1}, \tilde{\mathcal{E}}_{2}$ and $\left.p_{i n}\right)$ which is independent of $\gamma$ and $\theta$. Thus, the optimal placement is independent of scale parameter $\gamma$ and the aggregation parameter $\theta$.

Theorem 3 has important implications for market design. It says that the optimal intermediate market placement can be decided independently of how many wind farms are there in the system, and how correlated their outputs are. Thus, the system operator can keep the placement of this market fixed as more and more wind energy is incorporated into the system.

\section{The value of additional forward markets}

One of the key objectives of this paper is to study the impact of increased renewable penetration on the structure of electricity markets. As seen in the previous section, the optimal placement of the intermediate market is independent of the amount of wind present in the system. In this section, we look at another important market design question: can we facilitate the penetration of renewable energy by providing additional forward markets? It is commonly suggested that having additional markets would be beneficial, since this allows the utility to better exploit the evolution of the wind forecast. Indeed, the intuition is true in terms of procurement cost - having additional forward markets benefits the utility company (and thus the end consumer) by lowering the average cost of conventional energy procurement.

However, minimizing cost is not the only goal. Another important question is if additional forward markets also lower the total amount of conventional energy that a utility company needs to procure. From the environmental viewpoint, this is an extremely relevant question; reducing conventional energy use is one of the key driving factors for the renewable portfolio standards. Indeed, one would desire that the policy decision of adding a forward market for conventional energy increases the efficiency of the available renewable sources by decreasing our consumption of conventional generation.

In order to address the impact of additional markets on total procurement, we study the effect of the addition of a single intermediate (forward) market on the (average) total conventional procurement. Specifically, we compare the average total procurement under the three market scenario 
with the average total procurement under a scenario where there is no intermediate market. Let us denote the average total procurement under the three market scenario by $T P_{l, i, r}$, and let $T P_{l, r}$ represent the total procurement under the scenario with no intermediate market.

Intuitively, one expects that the addition of an intermediate market helps utility companies exploit the refined wind estimate to better match its residual demand with the overall conventional procurement. This would suggest that $\mathbb{E}\left[T P_{l, i, r}\right]<\mathbb{E}\left[T P_{l, r}\right]$; in other words, the addition of an intermediate market helps reduce total procurement. However, as the lemma below suggests, this intuition is not always correct. In fact, for some forecast evolution models and price structures, the addition of an intermediate forward market actually increases the average total procurement. This result is summarized in the Lemma below (the proof is provided in the Appendix).

Lemma 2. If the distribution of $\mathcal{E}_{2}$ has infinite support on the left (i.e., $L_{2}=-\infty$ ), and

$$
\lim _{x \rightarrow-\infty} \frac{f_{\mathcal{E}_{2}}(x)}{F_{\mathcal{E}_{2}}(x)}=0
$$

then, there exist values of demand $d$ and prices satisfying $0<p_{l t}<p_{i n}<p_{r t}$ such that

$$
\mathbb{E}\left[T P_{l, i, r}\right]>\mathbb{E}\left[T P_{l, r}\right]
$$

This lemma provides a counter example to the commonly held assumption that adding forward markets is always beneficial both from the cost and procurement viewpoints. It is important to note that the condition given in Equation (14) depends on the probability that the random variable $\mathcal{E}_{2}$ takes large negative values. Recall from Equation (2) that $\mathcal{E}_{2}=\hat{w}_{i n}-w$; in other words the condition in the lemma looks at the probability that the wind forecast at the intermediate time $\hat{w}_{i n}$ severely underestimates the actual wind generation.

We can interpret the condition in Equation (14) as a hazard rate condition. Note that if the distribution of $\mathcal{E}_{2}$ is symmetric, then the condition can be rewritten as

$$
\lim _{x \rightarrow \infty} \frac{f_{\mathcal{E}_{2}}(x)}{\bar{F}_{\mathcal{E}_{2}}(x)}=0
$$

The quantity in the above equation is the conventionally defined hazard rate function. Condition (14) states that the distribution of $\mathcal{E}_{2}$ is long-tailed to the left (Goldie and Klüppelberg 1998, Sigman 1999). Long-tailed distributions are an important sub-class of the class of heavy-tailed distributions, and are typically used to model phenomenon in which extreme values occur with a non-negligible probability. Examples of long-tailed distributions include distributions with powerlaw tails. Therefore, Lemma 2 can be interpreted informally as follows. If the wind estimate at 
the point of additional markets can significantly underestimate the wind generation $w$ with a non-negligible probability, then the average total procurement may increase with the additional market.

Lemma 2 highlights the importance of building better wind prediction models and understanding the distributions of wind prediction errors. The lemma also suggests that, if the probability that the wind forecast severely underestimates the actual available wind is small, then adding an intermediate might reduce the average total procurement. The following theorem formalizes this intuition by giving a sufficient condition for this to occur.

TheOREm 4. Suppose that the distribution of $\mathcal{E}_{2}$ satisfies the following properties:

(i) $f_{\mathcal{E}_{2}}(x)$ is non-decreasing for $x<0$ and non-increasing for $x>0$,

(ii) $\frac{f_{\mathcal{E}_{2}}(x)}{F_{\mathcal{E}_{2}}(x)}$ is strictly decreasing over $x \in\left(L_{2}, 0\right)$,

(iii) $\frac{f_{\mathcal{E}_{2}}^{\prime}(x)}{f_{\mathcal{E}_{2}}(x)}$ is non-increasing over $x \in\left(L_{2}, 0\right)$.

Then, under Assumption 1, $\mathbb{E}\left[T P_{l, i, r}\right]<\mathbb{E}\left[T P_{l, r}\right]$.

Theorem 4 highlights that there are a broad set of forecast error distributions for which the introduction of an intermediate market will lower the average total procurement. These are defined via three conditions. The first condition simply states that the density function $f_{\mathcal{E}_{2}}$ is unimodal at $x=0$, i.e., it has its maximum at $x=0$, and is monotone over $x<0$ and $x>0$. The last two conditions are concerned with the behavior of the distribution of $\mathcal{E}_{2}$ when $\mathcal{E}_{2} \leq 0$, i.e., when $\hat{w}_{\text {in }}$ under estimates the actual wind generation $w$. Condition $(i i)$ is in contrast with the condition in Lemma 2; it requires that the hazard rate of the left tail of $\mathcal{E}_{2}$ be strictly decreasing. This implies that $\mathcal{E}_{2}$ is light-tailed to the left. The conditions of Theorem 4 are satisfied by many common distributions, such as the zero mean Gaussian distribution and the zero mean uniform distribution.

\section{Related literature}

There are two distinct streams of literature related to the current problem.

The first stream of literature is that related to the classic newsvendor problem (Porteus 2002, Khouja 1999). Our optimal procurement formulation $(P)$ is mathematically equivalent to a variant of the newsvendor problem. Indeed, by substituting demand by the residual demand, i.e., demand minus wind (thereby shifting the uncertainty from the supply side to the demand side) and interpreting the real time price as a shortfall penalty, the optimization $(P)$ reduces to single period newsvendor problem in which procurements can be made at two different lead times. See Silver et al. (1998) for a survey of the literature relating to such variations of the newsvendor problem. In this space, the formulation in Wang et al. (2012) is closest to that in the current paper. However, 
in contrast to this body of work, the focus of the current paper is to understand the impact on electricity markets of the increasing supply side uncertainty induced by an increasing penetration of renewable resources. To the best of our knowledge, an effort to understand the effect of "scaling up the uncertainty" has not been attempted in the literature pertaining to the newsvendor problem.

The second stream of literature related to the current paper is work studying the introduction of intermittent renewable sources to electricity markets. Recently, there has been considerable research in this direction, for example, Meyn et al. (2010), Varaiya et al. (2011), Bitar et al. (2011), Rajagopal et al. (2012). Of particular note is the work in Meyn et al. (2010), which studies an electricity market equilibrium in the presence of wind generation, and the work in Bitar et al. (2011) and Cai et al. (2011), which analyzes the optimal bidding strategies for wind producers in the real time market. Kim and Powell (2011) study energy commitments made by wind producers in presence of electrical storage, while Korpaas et al. (2003) studies scheduling and operation of storage for wind power producers in electricity markets. The setting considered in this paper, in which the utility contracts on a long term basis the output of wind farms is also studied in Varaiya et al. (2011), Rajagopal et al. (2012). These papers also study optimal procurement strategies for the utility. In fact, the formulation $(P)$ in this paper is a special case of that in Varaiya et al. (2011). However, these papers focus primarily on the solving of the 'static' optimal procurement problem using techniques within framework of Markov decision processes. In contrast, the current paper focuses on the impact of increasing wind penetration and market structure (i.e., placement and inclusion/exclusion of markets). Additionally, the work in Varaiya et al. (2011), Rajagopal et al. (2012) focuses on numerical solutions for the optimal procurement while the current paper focuses on deriving closed form, interpretable, expressions for procurement that highlight the impact of the market structure.

\section{Concluding remarks}

Our goal in this paper is to quantify the impact of increasing penetration of intermittent, unpredictable, renewable energy on (i) the procurement of conventional generation by utilities, and (ii) the structure and efficiency of electricity markets. To accomplish this, we consider a three tiered model of electricity markets. Within this context, we derive the optimal strategy for procurement of conventional generation and analyze the changes to this strategy as the penetration of renewable increases. Additionally, we study the impact of increasing renewable penetration on the value and the placement of an intermediate electricity market. 
The main messages that follow from our results are the following. First, as is commonly recognized, reducing the dependence between the renewable sources (by locating different renewable sources geographically far apart) is key to ensuring efficient utilization of renewable generation. More surprisingly, it turns out that the optimal placement of the intermediate market does not change as renewable penetration increases. Further, the impact of the intermediate market on the total procurement can be either positive or negative depending on the the distribution of forecast errors. The results in this paper highlight that there are many important, and counterintuitive issues with respect to the incorporation of renewable generation into electricity markets.

This work extends easily along several directions. The model in our work can be easily extended to allow for an arbitrary number of forward markets for conventional energy procurement. Another extension that can be easily incorporated in our model is to allow utility companies to sell excess conventional generation in forward markets (as in Rajagopal et al. (2012)). In our model, we assumed that the exact realized wind energy is known at the time of the real time market. This assumption can also be relaxed by introducing a third distribution that reflects the error between wind forecasts in the real time market and the actual realized wind (as in Rajagopal et al. (2012)). The results presented in this paper can be easily generalized to this setting under the requirement that the utility company maintains a certain loss of load probability at the time of the real time market. There are, however, some non-trivial extensions to this model which are interesting opportunities for future research. One such extension is to understand the role of large quantities of grid energy storage on the optimal procurement policy of utility companies. Another extension of interest is to take into account ramp constraints of various generators, which would couple procurement decisions between different time instants.

More generally, the big question for the area is how and where renewables should be allowed to interact with electricity markets. The setting considered here is one extreme, where renewable energy only interacts through long-term contracts. The other extreme, where renewable energy interacts only in the real time market, is also studied in recent work. Hopefully, building on these results, we can move toward a unified approach highlighting the most efficient manner for renewable energy to interact with the electricity markets.

\section{Appendix A: Proof of Theorem 1.}

To compute the optimal procurement strategy, we first look at the decision in the real time. Recall that the optimal procurement in real time is a function of the actual available wind as well as the total conventional energy procured until that time. Let $q_{l t}$ and $q_{i n}$ be the quantities procured in 
long term and intermediate markets respectively. Note that the utility company needs to satisfy the demand. Thus, the optimal strategy in real time is to procure enough conventional energy to exactly meet the demand, i.e., $q_{r t}^{*}=\left[d-w-q_{l t}-q_{i n}\right]_{+}$. This real time procurement strategy ensures that the last constraint in the optimization problem is always satisfied.

Having decided the optimal strategy for real time, the optimization problem $(P)$ can now be re-written as

$$
\begin{aligned}
& \min _{q_{l t}, q_{i n}} \mathbb{E}\left[p_{l t} q_{l t}+p_{\text {in }} q_{\text {in }}+p_{r t}\left[d-w-q_{l t}-q_{i n}\right]_{+}\right] \\
& \text {subject to } q_{l t} \geq 0, q_{\text {in }} \geq 0 .
\end{aligned}
$$

This problem is a 3-stage Markov decision process (Bertsekas 2007), with the stages corresponding to the long term, intermediate, and real time markets. The state in each market is the tuple consisting of (i) the current wind estimate, and (ii) the total conventional generation procured so far. The action at each time corresponds to the procurement decisions at that time and the stage cost is the cost of that procurement. We summarize the Markov decision process associated with the optimal procurement problem in the table below.

\begin{tabular}{|l|l|l|l|}
\hline & Stage 1 & Stage 2 & terminal stage \\
\hline state & $\left(\hat{w}_{l t}, 0\right)$ & $\left(\hat{w}_{i n}, q_{l t}\right)$ & $\left(w, q_{l t}+q_{\text {in }}\right)$ \\
action & $q_{l t}$ & $q_{i n}$ & \\
disturbance & $\mathcal{E}_{1}$ & $\mathcal{E}_{2}$ & \\
stage cost & $p_{l t} q_{l t}$ & $p_{\text {in }} q_{i n}$ & $p_{r t}\left[d-w-q_{l t}-q_{i n}\right]_{+}$ \\
\hline
\end{tabular}

We can now compute the optimal procurement strategy for the intermediate and the long term markets using the dynamic programing algorithm Bertsekas (2007). In the intermediate market, the optimal procurement strategy is the minimizer of the expected cost to go; it is therefore the solution to the following optimization problem

$$
\min _{q_{i n} \geq 0} p_{i n} q_{i n}+\mathbb{E}_{\mathcal{E}_{2}}\left\{p_{r t}\left[d-w-q_{l t}-q_{i n}\right]_{+}\right\} .
$$

Using Equation (2) to write $w=\hat{w}_{i n}-\mathcal{E}_{2}$ and making the substitution $q_{i n}=d-q_{l t}-\hat{w}_{i n}+r$, we can write the objective function in the above minimization problem as

$$
\xi(r)=p_{i n}\left(d-q_{l t}-\hat{w}_{i n}+r\right)+p_{r t} \mathbb{E}_{\mathcal{E}_{2}}\left[\mathcal{E}_{2}-r\right]_{+} .
$$

Here, we can think of $r$ as the additional reserve required in the intermediate market. By direct differentiation, we get that

$$
\xi^{\prime}(r)=p_{i n}-p_{r t} \bar{F}_{\mathcal{E}_{2}}(r)
$$


Since $\xi^{\prime}(r)$ is non-decreasing, $\xi(\cdot)$ is convex. Moreover, $r_{i n} \in\left(L_{2}, R_{2}\right)$ is the unique minimizer of $\xi(\cdot)$ over $\mathbb{R}$. It is now easy to see that minimization (15) is convex, and that the optimal procurement in the intermediate market is given by (4).

The optimal cost to go from the intermediate stage onwards is given by

$$
J_{2}^{*}\left(\hat{w}_{i n}, q_{l t}\right)=p_{i n}\left[d-\hat{w}_{i n}-q_{l t}+r_{i n}\right]_{+}+\mathbb{E}_{\mathcal{E}_{2}}\left\{p_{r t}\left[d-\left(\hat{w}_{i n}-\mathcal{E}_{2}\right)-q_{l t}-\left[d-\hat{w}_{i n}-q_{l t}+r_{i n}\right]_{+}\right]_{+}\right\} .
$$

In the long term market, the optimal procurement is the solution to the following optimization.

$$
\min _{q_{l t} \geq 0} p_{l t} q_{l t}+\mathbb{E}_{\mathcal{E}_{1}} J_{2}^{*}\left(\hat{w}_{i n}, q_{l t}\right) .
$$

Using Equation (2) to write $\hat{w}_{i n}=\hat{w}_{l t}-\mathcal{E}_{1}$ and substituting Equation (16), we can write the objective function in the above minimization problem as

$$
\begin{aligned}
J\left(q_{l t}, \hat{w}_{l t}\right)=p_{l t} q_{l t}+ & \mathbb{E}_{\mathcal{E}_{1}}\left\{p_{i n}\left[d-\left(\hat{w}_{l t}-\mathcal{E}_{1}\right)-q_{l t}+r_{i n}\right]_{+}+\right. \\
& \left.\mathbb{E}_{\mathcal{E}_{2}}\left\{p_{r t}\left[d-\left(\hat{w}_{l t}-\mathcal{E}_{1}-\mathcal{E}_{2}\right)-q_{l t}-\left[d-\left(\hat{w}_{l t}-\mathcal{E}_{1}\right)-q_{l t}+r_{i n}\right]_{+}\right]_{+}\right\}\right\}
\end{aligned}
$$

We make the substitution $q_{l t}=d-\hat{w}_{l t}+r$, with the interpretation that $r$ is the additional reserve in the long term. We can now write the objective function as

$$
\begin{aligned}
\varphi(r)= & p_{l t}\left(d-\hat{w}_{l t}+r\right)+p_{i n} \mathbb{E}\left[\mathcal{E}_{1}-\left(r-r_{i n}\right)\right]_{+}+p_{r t} \mathbb{E}\left[\mathcal{E}_{1}+\mathcal{E}_{2}-r-\left[\mathcal{E}_{1}-\left(r-r_{i n}\right)\right]_{+}\right]_{+} \\
= & p_{l t}\left(d-\hat{w}_{l t}+r\right)+p_{i n} \mathbb{E}\left[\mathcal{E}_{1}-\left(r-r_{i n}\right)\right]_{+}+p_{r t} \mathbb{E}\left[\mathcal{E}_{2}-r+\min \left\{\mathcal{E}_{1}, r-r_{i n}\right\}\right]_{+} \\
= & p_{l t}\left(d-\hat{w}_{l t}+r\right)+p_{i n} \int_{r-r_{i n}}^{\infty}\left(y-\left(r-r_{i n}\right)\right) f_{\mathcal{E}_{1}}(y) d y \\
& \quad+p_{r t}\left(\bar{F}_{\mathcal{E}_{1}}\left(r-r_{i n}\right) \int_{r_{i n}}^{\infty}\left(z-r_{i n}\right) f_{\mathcal{E}_{2}}(z) d z+\int_{z=r}^{\infty} \int_{y=-\infty}^{r-r_{i n}}(z-r) f_{\mathcal{E}_{1}}(y) f_{\mathcal{E}_{2}}(z-y) d z d y\right) .
\end{aligned}
$$

Recall that the random variables $\mathcal{E}_{1}$ and $\mathcal{E}_{2}$ are defined over the support $\left(L_{1}, R_{1}\right)$ and $\left(L_{2}, R_{2}\right)$ respectively. Here, we interpret the density function to be zero outside the support of the random variables, if the support is finite.

Differentiating the above objective function yields

$$
\begin{aligned}
\varphi^{\prime}(r) & =p_{l t}-p_{i n} \bar{F}_{\mathcal{E}_{1}}\left(r-r_{i n}\right)-p_{l t} \int_{z=r}^{\infty} \int_{y=-\infty}^{r-r_{i n}} f_{\mathcal{E}_{1}}(y) f_{\mathcal{E}_{2}}(z-y) d z d y \\
& =h(r),
\end{aligned}
$$

where $h(r)$ is defined in Equation (7). We have

$$
\varphi^{\prime \prime}(r)=h^{\prime}(r)=f_{\mathcal{E}_{1}}\left(r-r_{i n}\right)\left(p_{i n}-p_{r t} \bar{F}_{\mathcal{E}_{2}}\left(r_{i n}\right)\right)+p_{r t} \int_{-\infty}^{r-r_{i n}} f_{\mathcal{E}_{1}}(y) f_{\mathcal{E}_{2}}(r-y) d y
$$




$$
=p_{r t} \int_{r_{i n}}^{\infty} f_{\mathcal{E}_{1}}(r-z) f_{\mathcal{E}_{2}}(z) d z .
$$

Since $\varphi^{\prime \prime}(\cdot)$ is non-negative, $\varphi(\cdot)$ is convex. Since $f_{\mathcal{E}_{i}}(x)>0$ over $x \in\left(L_{i}, R_{i}\right)$, it follows that $\varphi^{\prime \prime}(r)>0$ for $r \in\left(L_{1}+r_{i n}, R_{1}+R_{2}\right)$, and that $\varphi^{\prime \prime}(r)=0$ for all $r<L_{1}+r_{i n}$ and $r>R_{1}+R_{2}$. This implies that $\varphi^{\prime}(r)$ is strictly increasing over the range $r \in\left(L_{1}+r_{i n}, R_{1}+R_{2}\right)$. Note that

$$
\lim _{r \rightarrow-\infty} \varphi^{\prime}(r)=p_{l t}-p_{i n}<0,
$$

and

$$
\lim _{r \rightarrow \infty} \varphi^{\prime}(r)=p_{l t}>0
$$

It therefore follows that the equation $\varphi^{\prime}(r)=0$ has a unique solution $r_{l t} \in\left(L_{1}+r_{i n}, R_{1}+R_{2}\right)$. Moreover, $r_{l t}$ is the unique minimizer of $\varphi(\cdot)$ over $\mathbb{R}$. Finally, it follows as before that the optimization (17) is convex, and that the optimal procurement in the long term market is given by (3).

\section{Appendix B: Proofs of Theorem 4 and Lemma 2}

In this section, we prove Theorem 4 and Lemma 2. We first characterize the optimal procurement policy with no intermediate market in Section B.1. We then prove some preliminary lemmas in Section B.2. In Section B.3 we prove Theorem 4. Finally, we prove Lemma 2 in Section B.4.

\section{B.1. Optimal procurement policy with only long term and real time markets}

In this section, we analyze the optimal procurement strategy for the utility company when there is no intermediate market. Formally, this problem can be stated as follows.

$$
\begin{aligned}
& \min _{q_{l t}, q_{r t}} \mathbb{E}\left[p_{l t} q_{l t}+p_{r t} q_{r t}\right] \\
& \text { subject to } q_{l t} \geq 0, q_{r t} \geq 0 \\
& \quad q_{l t}+q_{r t}+w \geq d .
\end{aligned}
$$

Recall that in the above formulation, $q_{l t}$ and $q_{r t}$ are functions that depend on the corresponding wind estimate and the total quantities procured so far. The following lemma characterizes the optimal procurement policy for this case.

LEMma 3. The optimal procurement strategy for the utility company in the two tier market scenario given in problem $(\widetilde{P})$ is:

$$
\begin{gathered}
q_{l t}^{*}=\left[d-\hat{w}_{l t}+\bar{r}_{l t}\right]_{+}, \\
q_{r t}^{*}=\left[d-q_{l t}-w\right]_{+},
\end{gathered}
$$

where

$$
\bar{r}_{l t}:=\bar{F}_{\mathcal{E}_{1}+\mathcal{E}_{2}}^{-1}\left(\frac{p_{l t}}{p_{r t}}\right) .
$$

The lemma can be proved easily along the same lines as Theorem 1 and the proof is omitted. 


\section{B.2. Preliminary lemmas}

In the next two lemmas, we study the sensitivity of the optimal reserves and procurement with respect to the intermediate price $p_{i n}$. For the remainder of this section, we keep the distributions $\mathcal{E}_{1}$ and $\mathcal{E}_{2}$, as well as prices the $p_{l t}$ and $p_{r t}$ fixed. Before we proceed, we introduce the following additional notation. We define

$$
\bar{r}_{i n}=\bar{F}_{\mathcal{E}_{2}}^{-1}\left(\frac{p_{l t}}{p_{r t}}\right) .
$$

We can think of $\bar{r}_{i n}$ as the optimal reserve in a two market scenario, where the long term market occurs at the time $-T_{i n}$.

LEMma 4. For the optimal procurement problem $(P)$, the following results hold.

1. The intermediate reserve $r_{i n}$ is a strictly decreasing function of the intermediate price $p_{\text {in }}$ with

$$
\frac{\partial r_{i n}}{\partial p_{i n}}=-\frac{1}{p_{r t} f_{\mathcal{E}_{2}}\left(r_{i n}\right)}
$$

Furthermore,

$$
\begin{aligned}
& \lim _{p_{i n} \downarrow p_{l t}} r_{i n}=\bar{r}_{i n} \\
& \lim _{p_{i n} \uparrow p_{r t}} r_{i n}=L_{2} .
\end{aligned}
$$

2. The long term reserve $r_{l t}$ is a non-decreasing function of the intermediate price $p_{\text {in }}$ with

$$
\frac{\partial r_{l t}}{\partial p_{i n}}=\frac{\bar{F}_{\mathcal{E}_{1}}\left(r_{l t}-r_{i n}\right)}{p_{r t} \int_{-\infty}^{r_{l t}-r_{i n}} f_{\mathcal{E}_{1}}(y) f_{\mathcal{E}_{2}}\left(r_{l t}-y\right) d y} .
$$

Furthermore,

$$
\begin{aligned}
\lim _{p_{i n} \downarrow p_{l t}} r_{l t} & =L_{1}+\bar{r}_{i n}, \\
\lim _{p_{i n} \uparrow p_{r t}} r_{l t} & =\bar{r}_{l t}:=\bar{F}_{\mathcal{E}_{1}+\mathcal{E}_{2}}^{-1}\left(\frac{p_{l t}}{p_{r t}}\right)
\end{aligned}
$$

Proof. Equations (22) and (25) follow from an application of the implicit function theorem (Rudin 1976). Equations (23) and (24) follow easily from (6) and noting that fact that the random variable $\mathcal{E}_{2}$ is defined over the support $\left(L_{2}, R_{2}\right)$.

Note that $r_{l t}$ is a monotone function of the intermediate price $p_{l t}$. Hence, as $p_{i n} \downarrow p_{l t}$ or $p_{i n} \uparrow p_{r t}$, the long term reserve $r_{l t}$ has a limit. To prove Equation (26), suppose that as $p_{i n} \downarrow p_{l t}, r_{l t} \rightarrow \check{r}_{l t}$. From Equation (7), (by taking the limit $p_{i n} \downarrow p_{l t}$ ), we note that $\check{r}_{l t}$ is a solution of the equation

$$
\varphi(r):=p_{l t}-p_{l t} \bar{F}_{\mathcal{E}_{1}}\left(r-\bar{r}_{i n}\right)-p_{r t} \int_{z=r}^{\infty} \int_{y=-\infty}^{r-\bar{r}_{i n}} f_{\mathcal{E}_{1}}(y) f_{\mathcal{E}_{2}}(z-y) d z d y=0 .
$$

It is easy to check that 
1. $\varphi(r)=0$ for $r \leq L_{1}+\bar{r}_{i n}$,

2. For $r \in\left(L_{1}+\bar{r}_{i n}, R_{1}+R_{2}\right)$, we have

$$
\varphi^{\prime}(r)=p_{r t} \int_{r_{i n}}^{\infty} f_{\mathcal{E}_{1}}(r-z) f_{\mathcal{E}_{2}}(z) d z>0
$$

3. For $r>R_{1}+R_{2}, \varphi^{\prime}(r)=0$.

Thus we conclude that $\varphi(r)>0$ for $r>L_{1}+\bar{r}_{i n}$, which implies that $\check{r}_{l t} \leq L_{1}+\bar{r}_{i n}$. However, for any $p_{i n} \in\left(p_{l t}, p_{r t}\right)$, we have $r_{l t}>L_{1}+r_{i n}$, which implies that $\check{r}_{l t} \geq L_{1}+\bar{r}_{i n}$. Therefore, $\check{r}_{l t}=L_{1}+\bar{r}_{i n}$, which proves Equation (26).

To prove Equation (27), suppose that as $p_{i n} \uparrow p_{r t}, r_{l t} \rightarrow \hat{r}_{l t}$. Taking the limit as $p_{i n} \uparrow p_{r t}$ in Equation (7), we conclude that $\hat{r}_{l t}$ satisfies

$$
p_{l t}-p_{r t} P\left(\mathcal{E}_{1}>\hat{r}_{l t}-L_{2}\right)-p_{r t} P\left(\mathcal{E}_{1}+\mathcal{E}_{2}>\hat{r}_{l t} ; \mathcal{E}_{1} \leq \hat{r}_{l t}-L_{2}\right)=0
$$

Define $A$ to be the event that $\left\{\mathcal{E}_{1}+\mathcal{E}_{2}>\hat{r}_{l t}\right\}$ and $B$ to be the event that $\left\{\mathcal{E}_{1}>\hat{r}_{l t}-L_{2}\right\}$. Using the fact that $P\left(A \cap B^{c}\right)+P(B)=P(A \cup B)$, we get that $\hat{r}_{l t}$ satisfies the equation

$$
p_{l t}-p_{r t} P\left(\left\{\mathcal{E}_{1}+\mathcal{E}_{2}>\hat{r}_{l t}\right\} \cup\left\{\mathcal{E}_{1}>\hat{r}_{l t}-L_{2}\right\}\right)=0
$$

Note that the random variable $\mathcal{E}_{2} \in\left(L_{2}, R_{2}\right)$ and hence the event $\left\{\mathcal{E}_{1}>\hat{r}_{l t}-L_{2}\right\}$ implies the event $\left\{\mathcal{E}_{1}+\mathcal{E}_{2}>\hat{r}_{l t}\right\}$ almost surely. Thus, the long term reserve $\hat{r}_{l t}$ satisfies the equation

$$
p_{l t}-p_{r t} P\left(\mathcal{E}_{1}+\mathcal{E}_{2}>\hat{r}_{l t}\right)=0
$$

which implies Equation (27). This proves the lemma.

The next lemma studies how the total procurement changes as a function of the intermediate price $p_{l t}$. Recall that $\mathbb{E}\left[T P_{l, i, r}\right]$ and $\mathbb{E}\left[T P_{l, r}\right]$ are the expected total procurement under the three market scenario (long term, intermediate, and real time) as well as under the two markets (long term and real time) respectively. For the remainder of this section, we introduce some additional notation. We let $q_{l t ; l, i, r}^{*}, q_{i n ; l, i, r}^{*}$, and $q_{r t ; l, i, r}^{*}$ to the optimal procurement quantities in the long term, intermediate, and the real time market under the scenario that the utility company has three opportunities to procure energy. These optimal quantities are given in Theorem 1. Similarly, we let $q_{l t ; l, r}^{*}$ and $q_{r t ; l, r}^{*}$ to be the optimal quantities in the long term and in the real time under the scenario where there is no intermediate market. These quantities are given by the Lemma 3 . We have the following lemma. 
Lemma 5. As the intermediate price $p_{i n}$ approaches the real time price $p_{r t}$, the expected total procurement in three market scenario converges to the expected total procurement in the two market scenario. That is,

$$
\lim _{p_{i n} \uparrow p_{r t}} \mathbb{E}\left[T P_{l, i, r}\right]=\mathbb{E}\left[T P_{l, r}\right] .
$$

Proof. We prove this lemma using a sample path argument. A sample path is defined by the values of $\hat{w}_{l t}, \mathcal{E}_{1}$, and $\mathcal{E}_{2}$. We will show that as $p_{i n} \uparrow p_{r t}$,

$$
q_{l t ; l, i, r}^{*}+q_{i n ; l, i, r}^{*}+q_{r t ; l, i, r}^{*} \longrightarrow q_{l t ; l, r}^{*}+q_{r t ; l, r}^{*}
$$

almost surely. An elementary application of the dominated convergence theorem would then prove the lemma.

From Theorem 1, Lemma 4, and Lemma 3, it is easy to show that as $p_{i n} \uparrow p_{r t}$, we have

$$
q_{l t ; l, i, r}^{*} \longrightarrow q_{l t ; l, r}^{*}
$$

almost surely. Thus, to prove (28), it suffices to show that

$$
q_{i n ; l, i, r}^{*}+q_{r t ; l, i, r}^{*} \longrightarrow q_{r t ; l, r}^{*}
$$

almost surely as $p_{i n} \uparrow p_{r t}$. Recall that $q_{r t ; l, r}^{*}=\left[d-w-q_{l t ; l, r}^{*}\right]_{+}$From Lemma 4 and Equation (29), we conclude that as $p_{i n} \uparrow p_{r t}$. we have

$$
\begin{aligned}
& q_{i n ; l, i, r}^{*} \longrightarrow\left[d-\hat{w}_{i n}-q_{l t ; l, r}^{*}+L_{2}\right]_{+}, \\
& q_{r t ; l, i, r}^{*} \longrightarrow\left[d-w-q_{l t ; l, r}^{*}-\left[d-\hat{w}_{i n}+L_{2}\right]_{+}\right]_{+} .
\end{aligned}
$$

To see that Equation (31) implies (30), we consider the following two cases. On sample paths satisfying $d<\hat{w}_{i n}+q_{l t ; l, r}^{*}-L_{2}$, we have

$$
q_{i n ; l, i, r}^{*} \stackrel{p_{i n} \uparrow p_{r t}}{\longrightarrow} 0, \quad q_{r t ; l, i, r}^{*} \stackrel{p_{i n} \uparrow p_{r t}}{\longrightarrow}\left[d-w-q_{l t ; l, r}^{*}\right]_{+},
$$

which implies Equation (30). On sample paths satisfying $d \geq \hat{w}_{i n}+q_{l t ; l, r}^{*}-L_{2}$, we have

$$
q_{i n ; l, i, r}^{*} \stackrel{p_{i n} \uparrow p_{r t}}{\longrightarrow} d-\hat{w}_{i n}-q_{l t ; l, r}^{*}+L_{2}, \quad q_{r t ; l, i, r}^{*} \stackrel{p_{i n} \uparrow p_{r t}}{\longrightarrow}\left[\mathcal{E}_{2}-L_{2}\right]_{+} .
$$

Noting that $\mathcal{E}_{2} \geq L_{2}$ almost surely, we conclude that except possibly on a measure zero set of sample paths, we have

$$
q_{i n ; l, i, r}^{*}+q_{r t ; l, i, r}^{*} \stackrel{p_{i n} \uparrow p_{r t}}{\longrightarrow}\left(d-\hat{w}_{i n}-q_{l t ; l, r}^{*}+L_{2}\right)+\left(\mathcal{E}_{2}-L_{2}\right)=d-w-q_{l t ; l, r}^{*} .
$$

Since the procurements are always non-negative, the above equation implies Equation (30). This completes the proof. 
The above lemma states that the (optimal) total procurement in the three market scenario converges to that in the two market scenario (with only the long term and real time markets) as $p_{i n} \uparrow p_{r t} .$. Before we proceed further, we present an additional technical lemma.

Lemma 6. Suppose that the distribution of $\mathcal{E}_{2}$ satisfies the following properties:

(i) $f_{\mathcal{E}_{2}}(x)$ is non-decreasing for $x<0$ and non-increasing for $x>0$,

(ii) $\frac{f_{\mathcal{E}_{2}}(x)}{F_{\mathcal{E}_{2}}(x)}$ is strictly decreasing over $x \in\left(L_{2}, 0\right)$,

(iii) $\frac{f_{\mathcal{E}_{2}}^{\prime}(x)}{f_{\mathcal{E}_{2}}(x)}$ is non-increasing over $x \in\left(L_{2}, 0\right)$.

Then

$$
\left(\frac{p_{r t}-p_{l t}}{p_{r t}-p_{i n}}\right) f_{\mathcal{E}_{2}}\left(r_{i n}\right)>\int_{-\infty}^{r_{l t}-r_{i n}} f_{\mathcal{E}_{1}}(y) f_{\mathcal{E}_{2}}\left(r_{l t}-y\right) d y+\bar{F}_{\mathcal{E}_{1}}\left(r_{l t}-r_{i n}\right) f_{\mathcal{E}_{2}}\left(r_{i n}\right) .
$$

Proof. For this proof, we use the notation $\beta:=\bar{F}_{\mathcal{E}_{2}}(0)$. Let us also denote by $L\left(p_{i n}\right)$ and $R\left(p_{i n}\right)$ the left hand side and the right hand side of the Inequality (32) respectively. To prove the lemma, we need to show that $L\left(p_{i n}\right)>R\left(p_{i n}\right)$ for all values of $p_{i n} \in\left(p_{l t}, p_{r t}\right)$. Let us consider two separate cases.

Case 1: $p_{l t}<\beta p_{r t}$.

Consider first the case $p_{i n} \in\left(p_{l t}, \beta p_{r t}\right]$. For this case, we have $p_{i n} / p_{r t} \leq \beta$ and hence from Equation (6), we have that $r_{i n} \geq 0$. Using a change of variables, we can write $R\left(p_{i n}\right)$ as

$$
R\left(p_{i n}\right)=\int_{r_{i n}}^{\infty} f_{\mathcal{E}_{1}}\left(r_{l t}-y\right) f_{\mathcal{E}_{2}}(y) d y+\bar{F}_{\mathcal{E}_{1}}\left(r_{l t}-r_{i n}\right) f_{\mathcal{E}_{2}}\left(r_{i n}\right)
$$

Note that $f_{\mathcal{E}_{2}}(x)$ is non increasing for all $x>0$ and $r_{i n} \geq 0$. Thus, for all $y \geq r_{i n}$, we have $f_{\mathcal{E}_{2}}\left(r_{i n}\right) \geq$ $f_{\mathcal{E}_{2}}(y)$. Thus, we can write $R\left(p_{i n}\right)$ as

$$
\begin{aligned}
R\left(p_{i n}\right) & \leq f_{\mathcal{E}_{2}}\left(r_{i n}\right) \int_{r_{i n}}^{\infty} f_{\mathcal{E}_{1}}\left(r_{l t}-y\right) d y+\bar{F}_{\mathcal{E}_{1}}\left(r_{l t}-r_{i n}\right) f_{\mathcal{E}_{2}}\left(r_{i n}\right), \\
& =f_{\mathcal{E}_{2}}\left(r_{i n}\right), \\
& <L\left(p_{i n}\right),
\end{aligned}
$$

where the last inequality follows from that fact that $p_{l t}<p_{i n}$. Next consider the case where $p_{i n} \in$ $\left(\beta p_{r t}, p_{r t}\right)$. From Equation (6), we have that $r_{i n}<0$. Furthermore, from the conditions of the lemma, we have that $f_{\mathcal{E}_{2}}(y) \leq f_{\mathcal{E}_{2}}(0)$ for all $y \in \mathbb{R}$. Thus, we can write

$$
\begin{aligned}
R\left(p_{i n}\right) & =\int_{r_{i n}}^{\infty} f_{\mathcal{E}_{1}}\left(r_{l t}-y\right) f_{\mathcal{E}_{2}}(y) d y+\bar{F}_{\mathcal{E}_{1}}\left(r_{l t}-r_{i n}\right) f_{\mathcal{E}_{2}}\left(r_{i n}\right), \\
& \leq f_{\mathcal{E}_{2}}(0) \int_{r_{i n}}^{\infty} f_{\mathcal{E}_{1}}\left(r_{l t}-y\right) d y+\bar{F}_{\mathcal{E}_{1}}\left(r_{l t}-r_{i n}\right) f_{\mathcal{E}_{2}}(0), \\
& \leq f_{\mathcal{E}_{2}}(0) .
\end{aligned}
$$


Let us now look at $L\left(p_{i n}\right)$. We will construct a lower bound for $L\left(p_{i n}\right)$ for $p_{\text {in }} \in\left(\beta p_{r t}, p_{r t}\right)$. Note that $\bar{F}_{\mathcal{E}_{2}}\left(r_{i n}\right)=p_{i n} / p_{r t}$ and hence we can write $L\left(p_{i n}\right)$ as

$$
L\left(p_{i n}\right)=\left(\frac{p_{r t}-p_{l t}}{p_{r t}}\right) \frac{f_{\mathcal{E}_{2}}\left(r_{i n}\right)}{F_{\mathcal{E}_{2}}\left(r_{i n}\right)} .
$$

Recall that for $p_{i n} \in\left(\beta p_{r t}, p_{r t}\right)$, we have $r_{i n}<0$. Furthermore from Lemma $4, r_{i n}$ is strictly decreasing function of $p_{i n}$. From the statement of the lemma, $f_{\mathcal{E}_{2}}(x) / F_{\mathcal{E}_{2}}(x)$ is a strictly decreasing function for all $x \leq 0$. Hence, $L\left(p_{i n}\right)$ is strictly increasing over $p_{i n} \in\left(\beta p_{r t}, p_{r t}\right)$. Thus, we have

$$
\begin{aligned}
L\left(p_{i n}\right) & >L\left(\beta p_{r t}\right), \\
& =\left(\frac{p_{r t}-p_{l t}}{p_{r t}-\beta p_{r t}}\right) f_{\mathcal{E}_{2}}(0),
\end{aligned}
$$

where the last equality follows since $r_{i n}=0$ when $p_{i n}=\beta p_{r t}$. Since $p_{l t}<\beta p_{r t}$, the term in the parentheses in the above equation is greater than one. Hence $L\left(p_{i n}\right)>f_{\mathcal{E}_{2}}(0)$ and from Equation (33), it follows that $L\left(p_{i n}\right)>R\left(p_{i n}\right)$. This proves Case 1 .

Case 2: $p_{l t} \geq \beta p_{r t}$.

In this case, we note that $p_{i n} / p_{r t}>\beta$ and hence from Equation (6), we get that $r_{i n}<0$. Using the argument similar to Case 1 , it is easy to verify that $L\left(p_{i n}\right)$ is a strictly increasing function for $p_{\text {in }} \in\left(p_{l t}, p_{r t}\right)$. Thus, we have

$$
\begin{aligned}
L\left(p_{i n}\right) & >\lim _{p_{i n} \downarrow p_{l t}} L\left(p_{i n}\right), \\
& =f_{\mathcal{E}_{2}}\left(\bar{r}_{i n}\right),
\end{aligned}
$$

where we use the fact that as $p_{i n} \downarrow p_{l t}, r_{i n} \rightarrow \bar{r}_{i n}$ (Lemma 4). Thus, to prove the lemma for this case, it suffices to show that $R\left(p_{i n}\right) \leq f_{\mathcal{E}_{2}}\left(\bar{r}_{i n}\right)$. From Lemma 4 , we note that $\lim _{p_{i n} \downarrow p_{l t}} r_{l t}-r_{i n}=L_{1}$ and hence

$$
\lim _{p_{i n} \downarrow p_{l t}} R\left(p_{i n}\right)=f_{\mathcal{E}_{2}}\left(\bar{r}_{i n}\right) .
$$

Thus, to prove the lemma, it suffices to show that $R\left(p_{i n}\right)$ is non-increasing over the range $p_{i n} \in$ $\left(p_{l t}, p_{r t}\right)$. To show this, we note that

$$
\begin{aligned}
\frac{\partial R\left(p_{i n}\right)}{\partial p_{i n}} & =\frac{\partial}{\partial p_{i n}}\left(\int_{-\infty}^{r_{l t}-r_{i n}} f_{\mathcal{E}_{1}}(y) f_{\mathcal{E}_{2}}\left(r_{l t}-y\right) d y+\bar{F}_{\mathcal{E}_{1}}\left(r_{l t}-r_{i n}\right) f_{\mathcal{E}_{2}}\left(r_{i n}\right)\right) \\
& =\frac{\partial r_{l t}}{\partial r_{i n}} \int_{-\infty}^{r_{l t}-r_{i n}} f_{\mathcal{E}_{1}}(y) f_{\mathcal{E}_{2}}^{\prime}\left(r_{l t}-y\right) d y+\frac{\partial r_{i n}}{\partial p_{i n}} \bar{F}_{\mathcal{E}_{1}}\left(r_{l t}-r_{i n}\right) f_{\mathcal{E}_{2}}^{\prime}\left(r_{i n}\right), \\
& =\frac{\bar{F}_{\mathcal{E}_{1}}\left(r_{l t}-r_{i n}\right)}{p_{r t}}\left[\frac{\int_{-\infty}^{r_{l t}-r_{i n}} f_{\mathcal{E}_{1}}(y) f_{\mathcal{E}_{2}}^{\prime}\left(r_{l t}-y\right) d y}{\int_{-\infty}^{r_{l t}-r_{i n}} f_{\mathcal{E}_{1}}(y) f_{\mathcal{E}_{2}}\left(r_{l t}-y\right) d y}-\frac{f_{\mathcal{E}_{2}}^{\prime}\left(r_{i n}\right)}{f_{\mathcal{E}_{2}}\left(r_{i n}\right)}\right]
\end{aligned}
$$




$$
=\frac{\bar{F}_{\mathcal{E}_{1}}\left(r_{l t}-r_{i n}\right)}{p_{r t}}\left[\frac{\int_{r_{i n}}^{\infty} f_{\mathcal{E}_{1}}\left(r_{l t}-y\right) f_{\mathcal{E}_{2}}^{\prime}(y) d y}{\int_{r_{i n}}^{\infty} f_{\mathcal{E}_{1}}\left(r_{l t}-y\right) f_{\mathcal{E}_{2}}(y) d y}-\frac{f_{\mathcal{E}_{2}}^{\prime}\left(r_{i n}\right)}{f_{\mathcal{E}_{2}}\left(r_{i n}\right)}\right] .
$$

Here the third equality follows from Lemma 4 and the last equality follows from a change of variable. We will show that $\frac{\partial R\left(p_{i n}\right)}{\partial p_{i n}} \leq 0$ by considering the following two separate cases.

Case 2a: Consider the case

$$
\int_{r_{i n}}^{0} f_{\mathcal{E}_{1}}\left(r_{l t}-y\right) f_{\mathcal{E}_{2}}(y) d y=0
$$

Recall that the density functions $f_{\mathcal{E}_{1}}(\cdot)$ and $f_{\mathcal{E}_{2}}(\cdot)$ are assumed to be strictly positive over the interior of the supports of $\mathcal{E}_{1}$ and $\mathcal{E}_{2}$ respectively. Therefore, the condition (35) implies that the intersection of the supports of the functions $f_{\mathcal{E}_{2}}(y)$ and $f_{\mathcal{E}_{1}}\left(r_{l t}-y\right)$ and $\left[r_{i n}, 0\right]$ has measure zero. This implies that

$$
\int_{r_{i n}}^{0} f_{\mathcal{E}_{1}}\left(r_{l t}-y\right) f_{\mathcal{E}_{2}}^{\prime}(y) d y=0
$$

Using (35) and (36), the expression for $\frac{\partial R\left(p_{i n}\right)}{\partial p_{i n}}$ in (34) can be rewritten as

$$
\frac{\partial R\left(p_{i n}\right)}{\partial p_{i n}}=\frac{\bar{F}_{\mathcal{E}_{1}}\left(r_{l t}-r_{i n}\right)}{p_{r t}}\left[\frac{\int_{0}^{\infty} f_{\mathcal{E}_{1}}\left(r_{l t}-y\right) f_{\mathcal{E}_{2}}^{\prime}(y) d y}{\int_{0}^{\infty} f_{\mathcal{E}_{1}}\left(r_{l t}-y\right) f_{\mathcal{E}_{2}}(y) d y}-\frac{f_{\mathcal{E}_{2}}^{\prime}\left(r_{i n}\right)}{f_{\mathcal{E}_{2}}\left(r_{i n}\right)}\right] \leq 0 .
$$

The inequality above follows from the assumption that $f_{\mathcal{E}_{2}}^{\prime}(x) \leq 0$ for $x \geq 0$.

Case 2b: We now consider the case

$$
\int_{r_{i n}}^{0} f_{\mathcal{E}_{1}}\left(r_{l t}-y\right) f_{\mathcal{E}_{2}}(y) d y>0
$$

Since $f_{\mathcal{E}_{2}}^{\prime}(x) \leq 0$ for $x \geq 0$,

$$
\int_{r_{i n}}^{\infty} f_{\mathcal{E}_{1}}\left(r_{l t}-y\right) f_{\mathcal{E}_{2}}^{\prime}(y) d y \leq \int_{r_{i n}}^{0} f_{\mathcal{E}_{1}}\left(r_{l t}-y\right) f_{\mathcal{E}_{2}}^{\prime}(y) d y
$$

Therefore, the expression for $\frac{\partial R\left(p_{i n}\right)}{\partial p_{i n}}$ in (34) can be bounded above as follows.

$$
\frac{\partial R\left(p_{i n}\right)}{\partial p_{i n}} \leq \frac{\bar{F}_{\mathcal{E}_{1}}\left(r_{l t}-r_{i n}\right)}{p_{r t}}\left[\frac{\int_{r_{i n}}^{0} f_{\mathcal{E}_{1}}\left(r_{l t}-y\right) f_{\mathcal{E}_{2}}^{\prime}(y) d y}{\int_{r_{i n}}^{0} f_{\mathcal{E}_{1}}\left(r_{l t}-y\right) f_{\mathcal{E}_{2}}(y) d y}-\frac{f_{\mathcal{E}_{2}}^{\prime}\left(r_{i n}\right)}{f_{\mathcal{E}_{2}}\left(r_{i n}\right)}\right] .
$$

Define

$$
\begin{aligned}
p(y) & =\frac{f_{\mathcal{E}_{1}}\left(r_{l t}-y\right)}{\int_{r_{i n}}^{0} f_{\mathcal{E}_{1}}\left(r_{l t}-y\right) d y}, \\
\xi(y) & =\frac{f_{\mathcal{E}_{2}}^{\prime}(y)}{f_{\mathcal{E}_{2}}(y)}, \quad \text { and } \\
\varphi(y) & =f_{\mathcal{E}_{2}}(y) .
\end{aligned}
$$


Note that $p(y)$ is a valid probability density function defined over the interval $\left(r_{i n}, 0\right)$. We can then write

$$
\frac{\partial R\left(p_{i n}\right)}{\partial p_{i n}}=\frac{\bar{F}_{\mathcal{E}_{1}}\left(r_{l t}-r_{i n}\right)}{p_{r t}}\left[\frac{\int_{r_{i n}}^{0} p(y) \xi(y) \varphi(y) d y}{\int_{r_{i n}}^{0} p(y) \varphi(y) d y}-\frac{f_{\mathcal{E}_{2}}^{\prime}\left(r_{i n}\right)}{f_{\mathcal{E}_{2}}\left(r_{i n}\right)}\right]
$$

Using Lemma 7, we get

$$
\begin{aligned}
\frac{\partial R\left(p_{i n}\right)}{\partial p_{i n}} & \leq \frac{\bar{F}_{\mathcal{E}_{1}}\left(r_{l t}-r_{i n}\right)}{p_{r t}}\left[\int_{r_{i n}}^{0} p(y) \frac{f_{\mathcal{E}_{2}}^{\prime}(y)}{f_{\mathcal{E}_{2}}(y)} d y-\frac{f_{\mathcal{E}_{2}}^{\prime}\left(r_{i n}\right)}{f_{\mathcal{E}_{2}}\left(r_{i n}\right)}\right], \\
& \leq 0
\end{aligned}
$$

where the last inequality follows from the fact that $f_{\mathcal{E}_{2}}^{\prime}(y) / f_{\mathcal{E}_{2}}(y)$ is non-increasing over the range $y \in\left(r_{i n}, 0\right)$.

We have now proved that $R\left(p_{i n}\right)$ is non-increasing over the range $p_{i n} \in\left(p_{l t}, p_{r t}\right)$. This completes the proof of Case 2.

The above lemma uses the following technical lemma.

Lemma 7. Let $[a, b]$ denote an interval on the real line. Suppose that $\xi:[a, b] \rightarrow \mathbb{R}$ is non-increasing, and $\varphi:[a, b] \rightarrow \mathbb{R}$ is non-decreasing. Also, suppose that $p(\cdot)$ is a probability density function defined over the interval $[a, b]$. Then

$$
\int_{a}^{b} p(y) \xi(y) \varphi(y) d y \leq\left[\int_{a}^{b} p(y) \xi(y) d y\right]\left[\int_{a}^{b} p(y) \varphi(y) d y\right] .
$$

To prove the above lemma, we look at the discrete version of the above inequality. The above lemma then follows naturally by taking a limit of Reimann sums.

LEMma 8. Consider finite sequences $\left\{\xi_{i}\right\}_{i=1}^{n},\left\{\varphi_{i}\right\}_{i=1}^{n}$, and $\left\{p_{i}\right\}_{i=1}^{n}$, satisfying

1. $\varphi_{i}$ is non-decreasing,

2. $\xi_{i}$ is non-increasing,

3. $p_{i} \geq 0, \sum_{i=1}^{n} p_{i}=1$.

Then

$$
\sum_{i} p_{i} \xi_{i} \varphi_{i} \leq\left[\sum_{i} p_{i} \xi_{i}\right]\left[\sum_{i} p_{i} \varphi_{i}\right]
$$

Proof. We can write the right hand side of the above inequality as

$$
\left[\sum_{i} p_{i} \xi_{i}\right]\left[\sum_{i} p_{i} \varphi_{i}\right]=\sum_{i} p_{i}^{2} \xi_{i} \varphi_{i}+\sum_{i, j: i \neq j} p_{i} p_{j} \xi_{i} \varphi_{j} .
$$

Thus to prove the lemma, it suffices to show that

$$
\sum_{i} p_{i} \xi_{i} \varphi_{i} \leq \sum_{i} p_{i}^{2} \xi_{i} \varphi_{i}+\sum_{i, j: i \neq j} p_{i} p_{j} \xi_{i} \varphi_{j}
$$


which is equivalent to proving that

$$
\sum_{i} p_{i}\left(1-p_{i}\right) \xi_{i} \varphi_{i} \leq \sum_{i, j: i \neq j} p_{i} p_{j} \xi_{i} \varphi_{j}
$$

Note that $\left(1-p_{i}\right)=\sum_{j \neq i} p_{j}$ and hence the above inequality is equivalent to

$$
\sum_{i, j: j \neq i} p_{i} p_{j} \xi_{i} \varphi_{i} \leq \sum_{i, j: i \neq j} p_{i} p_{j} \xi_{i} \varphi_{j}
$$

To prove the last inequality above, it suffices to check that for $i \neq j, \xi_{i} \varphi_{i}+\xi_{j} \varphi_{j} \leq \xi_{i} \varphi_{j}+\xi_{j} \varphi_{i}$. This inequality is equivalent to checking that for $i \neq j,\left(\xi_{i}-\xi_{j}\right)\left(\varphi_{i}-\varphi_{j}\right) \leq 0$ which follows from the monotonicity assumption on the sequences $\left\{\xi_{i}\right\}_{i=1}^{n},\left\{\varphi_{i}\right\}_{i=1}^{n}$. This completes the proof.

\section{B.3. Proof of Theorem 4}

Let $p_{l t}, p_{i n}$ and $p_{r t}$ be the prices in the three markets. Let $\Gamma(p)$ be the expected optimal total procurement in three markets, when the long term price is $p_{l t}$, the real time price is $p_{r t}$, and the intermediate price is $p$. In other words, $\Gamma(p)$ gives the expected total procurement for a variable intermediate price (denoted by $p$ ). Clearly, we have $\mathbb{E}\left[T P_{l, i, r}\right]=\Gamma\left(p_{i n}\right)$. From Lemma 5 , we know that

$$
\mathbb{E}\left[T P_{l, r}\right]=\lim _{p \uparrow p_{r t}} \Gamma(p) .
$$

Thus, to prove the theorem it suffices to show that $\Gamma(p)$ is a strictly increasing function of $p$. This would imply that

$$
\mathbb{E}\left[T P_{l, i, r}\right]=\Gamma\left(p_{\text {in }}\right)<\lim _{p \uparrow p_{r t}} \Gamma(p)=\mathbb{E}\left[T P_{l, r}\right] .
$$

From the statement of the theorem, Assumption 1 holds for prices $p_{l t}, p_{i n}$ and $p_{r t}$. From Lemma 4, we know that $r_{l t}$ is a non decreasing function of intermediate price. Hence for all $p \geq p_{i n}$, Assumption 1 holds. Thus, the optimal procurements for all intermediate price $p \geq p_{\text {in }}$ are given by Equation 8. Let $q_{l t}^{*}(p), q_{i n}^{*}(p)$, and $q_{r t}^{*}(p)$ be the optimal procurements for the intermediate price $p$. Thus, we have

$$
\begin{aligned}
\frac{\partial \mathbb{E}\left[q_{l t}^{*}(p)\right]}{\partial p} & =\frac{\partial}{\partial p}\left(d-\hat{w}_{l t}+r_{l t}\right) \\
& =\frac{\partial r_{l t}}{\partial p} . \\
\frac{\partial \mathbb{E}\left[q_{i n}^{*}(p)\right]}{\partial p} & =\frac{\partial}{\partial p} \mathbb{E}\left[\mathcal{E}_{1}-\left(r_{l t}-r_{i n}\right)\right]_{+} \\
& =\bar{F}_{\mathcal{E}_{1}}\left(r_{l t}-r_{i n}\right)\left(\frac{\partial r_{i n}}{\partial p}-\frac{\partial r_{l t}}{\partial p}\right) . \\
\frac{\partial \mathbb{E}\left[q_{r t}^{*}(p)\right]}{\partial p} & =\frac{\partial}{\partial p} \mathbb{E}\left[\mathcal{E}_{2}-r_{l t}+\min \left\{\mathcal{E}_{1}, r_{l t}-r_{i n}\right\}\right]_{+}
\end{aligned}
$$




$$
\begin{aligned}
& =\frac{\partial}{\partial p}\left(\bar{F}_{\mathcal{E}_{1}}\left(r_{l t}-r_{i n}\right) \int_{r_{i n}}^{\infty}\left(z-r_{i n}\right) f_{\mathcal{E}_{2}}(z) d z+\int_{z=r_{l t}}^{\infty} \int_{y=-\infty}^{r_{l t}-r_{i n}}\left(z-r_{l t}\right) f_{\mathcal{E}_{1}}(y) f_{\mathcal{E}_{2}}(z-y) d z d y\right) \\
& =-\bar{F}_{\mathcal{E}_{1}}\left(r_{l t}-r_{i n}\right) \bar{F}_{\mathcal{E}_{2}}\left(r_{i n}\right) \frac{\partial r_{i n}}{\partial p}-\frac{\partial r_{l t}}{\partial p} \int_{z=r_{l t}}^{\infty} \int_{y=-\infty}^{r_{l t}-r_{i n}}\left(z-r_{l t}\right) f_{\mathcal{E}_{1}}(y) f_{\mathcal{E}_{2}}(z-y) d z d y .
\end{aligned}
$$

The last step above follows from a direct differentiation. Combining the above equations, we have

$$
\begin{aligned}
\frac{\partial \Gamma(p)}{\partial p}=\frac{\partial r_{l t}}{\partial p}\left[1-\bar{F}_{\mathcal{E}_{1}}\left(r_{l t}-r_{i n}\right)-\int_{z=r_{l t}}^{\infty} \int_{y=-\infty}^{r_{l t}-r_{i n}}\left(z-r_{l t}\right) f_{\mathcal{E}_{1}}(y) f_{\mathcal{E}_{2}}(z-y) d z d y\right] \\
+\frac{\partial r_{l t}}{\partial p}\left[\bar{F}_{\mathcal{E}_{1}}\left(r_{l t}-r_{i n}\right)-\bar{F}_{\mathcal{E}_{1}}\left(r_{l t}-r_{i n}\right) \bar{F}_{\mathcal{E}_{2}}\left(r_{i n}\right)\right] \\
=\frac{\partial r_{l t}}{\partial p}\left[1-\frac{p_{l t}}{p_{r t}}-\left(1-\frac{p}{p_{r t}}\right) \bar{F}_{\mathcal{E}_{1}}\left(r_{l t}-r_{i n}\right)\right]+\frac{\partial r_{l t}}{\partial p} \bar{F}_{\mathcal{E}_{1}}\left(r_{l t}-r_{i n}\right)\left(1-\frac{p}{p_{r t}}\right) .
\end{aligned}
$$

The last equality follows from the fact that $h\left(r_{l t}\right)=0$ and that $\bar{F}\left(r_{i n}\right)=p / p_{r t}$. Substituting $\partial r_{l t} / \partial p$ and $\partial r_{i n} / \partial p$ from Lemma 4 and after some algebraic manipulation, we get that $\partial \Gamma(p) / \partial p>0$ if and only if

$$
\left(\frac{p_{r t}-p_{l t}}{p_{r t}-p}\right) f_{\mathcal{E}_{2}}\left(r_{i n}\right)>\int_{-\infty}^{r_{l t}-r_{i n}} f_{\mathcal{E}_{1}}(y) f_{\mathcal{E}_{2}}\left(r_{l t}-y\right) d y+\bar{F}_{\mathcal{E}_{1}}\left(r_{l t}-r_{i n}\right) f_{\mathcal{E}_{2}}\left(r_{i n}\right) .
$$

From Lemma 6, we know that this inequality holds for all $p \in\left[p_{i n}, p_{r t}\right)$. This completes the proof.

\section{B.4. Proof of Lemma 2.}

Let us fix some long term and real time market prices $p_{l t}$ and $p_{r t}$ such that $0<p_{l t}<p_{r t}$. Let $\Gamma(p)$ be the expected optimal total procurement in a three market scenario with long term price $p_{l t}$, real time price $p_{r t}$, and the intermediate price $p$. From Lemma 5, we know that

$$
\lim _{p \uparrow p_{r t}} \Gamma(p)=\mathbb{E}\left[T P_{l, r}\right] .
$$

So to prove the lemma, it suffices to show that there exists $p_{i n} \in\left(p_{l t}, p_{r t}\right)$ such that for all intermediate market price $p \in\left(p_{i n}, p_{r t}\right)$,

$$
\frac{\partial \Gamma(p)}{\partial p}<0
$$

This would imply that for all $p \in\left(p_{i n}, p_{r t}\right), \Gamma\left(p_{i n}\right)>\lim _{p \uparrow p_{r t}} \Gamma(p)=\mathbb{E}\left[T P_{l, r}\right]$. Since $\Gamma\left(p_{\text {in }}\right)=$ $\mathbb{E}\left[T P_{l, i, r}\right]$, the expected total procurement in three market scenario under the intermediate price $p_{i n}$, this implies a tuple of prices satisfying the claim of the lemma.

Pick $\bar{p} \in\left(p_{l t}, p_{r t}\right)$. Now choose demand $d$ large enough such that Assumption 1 holds when the intermediate price $p=\bar{p}$. From Lemma 4 , we know that $r_{l t}$ is a non decreasing function of intermediate price $p$. Thus, Assumption 1 continues to hold for all intermediate price $p \geq \bar{p}$. Following the argument similar to the proof of Theorem 4 , we can show that for all $p \in\left[\bar{p}, p_{r t}\right)$,

$$
\frac{\partial \Gamma(p)}{\partial p}<0
$$


if and only if

$$
\left(\frac{p_{r t}-p_{l t}}{p_{r t}-p}\right) f_{\mathcal{E}_{2}}\left(r_{i n}\right)<\int_{-\infty}^{r_{l t}-r_{i n}} f_{\mathcal{E}_{1}}(y) f_{\mathcal{E}_{2}}\left(r_{l t}-y\right) d y+\bar{F}_{\mathcal{E}_{1}}\left(r_{l t}-r_{i n}\right) f_{\mathcal{E}_{2}}\left(r_{i n}\right) .
$$

Let us denote by $L(p)$ and $R(p)$ the left hand side and the right hand side of the above inequality respectively. From Equation (6), we have that

$$
L(p)=\left(\frac{p_{r t}-p_{l t}}{p_{r t}}\right) \frac{f_{\mathcal{E}_{2}}\left(r_{i n}\right)}{F_{\mathcal{E}_{2}}\left(r_{i n}\right)} .
$$

From Lemma 4, we know that $\lim _{p \uparrow p_{r t}} r_{i n}=-\infty$. From the conditions of the lemma, this implies that

$$
\lim _{p \uparrow p_{r t}} L(p)=0 .
$$

Now let us look at $R(p)$. From Lemma 4 , note that $\lim _{p \uparrow p_{r t}}\left(r_{l t}-r_{i n}\right)=\infty$ and $\lim _{p \uparrow p_{r t}} r_{l t}=\bar{r}_{l t}$. Thus, using dominated convergence theorem, we get that

$$
\lim _{p \uparrow p_{r t}} R(p)=f_{\mathcal{E}_{1}+\mathcal{E}_{2}}\left(\bar{r}_{l t}\right)>0 .
$$

From Equation (38) and Equation (39), we conclude that there exists $p_{i n} \in\left(\bar{p}, p_{r t}\right)$ such that for all $p \in\left(p_{i n}, p_{r t}\right)$ the inequality in Equation (37) holds. This completes the proof.

\section{References}

Arrow, K.J., T. Harris, J. Marschak. 1951. Optimal inventory policy. Econometrica: Journal of the Econometric Society 250-272.

Baldovin, F., A.L. Stella. 2007. Central limit theorem for anomalous scaling due to correlations. Physical Review E 75(2).

Bertsekas, Dimitri P. 2007. Dynamic Programming and Optimal Control, Volume II. Athena Scientific, Nashua, New Hampshire.

Bitar, E., R. Rajagopal, P. Khargonekar, K. Poolla, P. Varaiya. 2011. "Bringing wind energy to market". Submitted to IEEE Transactions on Power Systems .

Cai, D.W.H., S. Adlakha, K.M. Chandy. 2011. Optimal contract for wind power in day-ahead electricity markets. IEEE Conference on Decision and Control.

CAISO. 2011. California independent service operator market processes URL http://www.caiso.com/ market/Pages/MarketProcesses . aspx.

DeMeo, E. A., G. A. Jordan, C. Kalich, J. King, M. R. Milligan, C. Murley, B. Oakleaf, M. J. Schuerger. 2007. "Accommodating wind's natural behavior". IEEE Power and Energy Magazine 5(6) 59-67.

GE Energy. 2010. Western Wind and Solar Integration Study. Tech. rep., National Renewable Energy Laboratory (NREL), Golden, CO. 
GE Energy Consulting. 2007. "Intermittency analysis project: appendix B. Impact of intermittent generation on operation of california power grid". PIER Final Project Report, CEC-500-2007-081-APB .

Global Wind Energy Council. 2009. Global Wind 2009 Report. Renewable Energy House, Brussels, Belgium

Goldie, C. M., C. Klüppelberg. 1998. Subexponential distributions. A Practical Guide to Heavy Tails: Statistical Techniques and Applications 435-459.

Graves, S.C., H.C. Meal, S. Dasu, Y. Qui. 1985. Two-stage production planning in a dynamic environment. Massachusetts Institute of Technology, Alfred P. Sloan School of Management.

Harris, C. 2006. Electricity Markets: Pricing, Structures and Economics (The Wiley Finance Series). Wiley.

Hausman, W.H. 1969. Sequential decision problems: A model to exploit existing forecasters. Management Science 93-111.

Heath, D.C., P.L. Jackson. 1994. Modeling the evolution of demand forecasts with application to safety stock analysis in production/distribution systems. IIE Transactions-Industrial Engineering Research and Development 26(3) 17-30.

Khouja, M. 1999. The single-period (news-vendor) problem: literature review and suggestions for future research. Omega $\mathbf{2 7}(5)$ 537-553.

Kim, J. H., W. B. Powell. 2011. Optimal energy commitments with storage and intermittent supply. Operations Research 59(6) 1347-1360.

Kirschen, D. S., G. Strbac. 2004. Fundamentals of Power System Economics. Wiley.

Korpaas, M., A. T. Holem, R. Hildrum. 2003. Operation and sizing of energy storage for wind power plants in a market system. International Journal of Electrical Power and Energy Systems 25(8) 599-606.

Meyn, S., M. Negrete-Pincetic, G. Wang, A. Kowli, E. Shafieepoorfard. 2010. The value of volatile resources in electricity markets. IEEE Conference on Decision and Control. 1029-1036.

Negrete-Pincetic, M., G. Wang, A. Kowli, H. Pulgar-Painemal. 2010. "Emerging issues due to the integration of wind power in competitive electricity markets". Power and Energy Conference at Illinois (PECI), 2010. 45-50.

Porteus, E. L. 2002. Foundations of Stochastic Inventory Theory. Stanford Business Books.

Rajagopal, R., E. E. Bitar, F. Wu, Varaiya. 2012. Risk limiting dispatch of wind power. Under submission.

Rudin, W. 1976. Principles of mathematical analysis, vol. 3. McGraw-Hill New York.

Sigman, K. 1999. A primer on heavy-tailed distributions. Queueing Syst. Theory Appl. 33(1-3) 261-275.

Silver, E.A., D.F. Pyke, R. Peterson, et al. 1998. Inventory management and production planning and scheduling, vol. 3. Wiley New York.

Southern California Edison. 2011. Southern california edison renewable energy URL http://www.sce.com/ PowerandEnvironment/Renewables/default.htm. 
Stoft, S. 2002. Power System Economics: Designing Markets for Electricity. Wiley-IEEE Press.

Umarov, S., C. Tsallis, S. Steinberg. 2008. On a q-central limit theorem consistent with nonextensive statistical mechanics. Milan journal of mathematics 76(1) 307-328.

Varaiya, P.P., F.F. Wu, J.W. Bialek. 2011. Smart operation of smart grid: Risk-limiting dispatch. Proceedings of the IEEE 99(1) 40-57.

Wang, T., A. Atasu, M. Kurtulus. 2012. A multi-ordering newsvendor model with dynamic forecast evolution. Under submission .

Wiser, R. 2008. Renewable Portfolio Standards in the United States - A Status Report with Data Through 2007. Lawrence Berkeley National Laboratory, LBNL Paper LBNL-154E . 